\title{
Extended GRA Method Based on Cumulative Prospect Theory with Probabilistic Hesitant Fuzzy Information for MAGDM and its Application to Green Supplier Selection
}

Ningna Liao

Sichuan Normal University - Chenglong Campus

Guiwu Wei ( $\nabla$ weiguiwu@163.com )

Sichuan Normal University https://orcid.org/0000-0001-9074-2005

\section{Xudong Chen}

Southwestern University of Finance and Economics

\section{Research Article}

Keywords: multiple attributes group decision making (MAGDM), probabilistic hesitant fuzzy sets (PHFS), cumulative prospect theory (CPT), grey relational analysis (GRA) method, Entropy.

Posted Date: July 12th, 2021

DOl: https://doi.org/10.21203/rs.3.rs-516991/v1

License: (c) (1) This work is licensed under a Creative Commons Attribution 4.0 International License.

Read Full License 


\title{
Extended GRA method Based on Cumulative Prospect Theory with Probabilistic Hesitant Fuzzy information for MAGDM and its Application to Green Supplier Selection
}

\author{
Ningna Liao ${ }^{1}$, Guiwu $\mathrm{Wei}^{{ }^{1 *}}$, Xudong Chen ${ }^{2}$ \\ ${ }^{1}$ School of Business, Sichuan Normal University, Chengdu, 610101, P.R. China \\ ${ }^{2}$ School of Accounting, Southwestern University of Finance and Economics, Chengdu, \\ 611130, P.R. China \\ *Corresponding author, E-mail:weiguiwu@163.com
}

\begin{abstract}
An extended grey relational analysis (GRA) method is introduced in this article to reduce the limitations of the classical GRA method using the cumulative prospect theory (CPT) which takes into account psychological factors such as the risk appetite of decision makers. Moreover, the circumstance of probabilistic hesitant fuzzy (PHF) which assigns probabilistic values to DMs' different levels of hesitation shows its superiority when making decisions in a complex environment. Meanwhile the weighting vector of each attribute is calculated according to the entropy which is calculated by the different prospect decision elements. Thus, in this paper, we proposed an extended GRA method based on cumulative prospect theory in the probabilistic hesitant fuzzy circumstance and applying the model in the selection of the green supplier. At last, the comparative analysis and the simulation analysis are made to show the practicability of this newly proposed method.
\end{abstract}

Key words: multiple attributes group decision making (MAGDM); probabilistic hesitant fuzzy sets (PHFS); cumulative prospect theory (CPT); grey relational analysis (GRA) method; Entropy.

\section{Introduction}

Multi-attribute decision making (MADM), as a branch of the multi-criteria decision making (MCDM), has discrete decision variables and a limited number of options which are evaluated[15]. To solve many MADM issues, a sea of decision-making methods has been developed over the past few decades. The more important ones, for example, like VIKOR (a multi-criteria compromise solution ordering method)method [6-8], MABAC (the multi-attribute border approximation area comparison) method [9-12], CODAS(combinative distance based assessment) method[13-16] EDAS 
(the evaluation based on distance from average solution) method [17-22], MOORA (the multiobjective optimization ratio analysis)method [23-26], TODIM method[27-30] and Taxonomy method[31,32] and so on. These methods each have different advantages and disadvantages due to their calculation principles and among them, the GRA (grey relational analysis) method which is the part of the grey system theory introduced by Deng [33] and now it is widely applied in many fields. The grey system theory includes the GRA method is introduced to solve the issues under a complex environment with the unknown information. Specially, the GRA method is appropriate for solving the issues which is with complicated interrelationships between multiple factors and variables( Kuo, Yang and Huang [34]), and the specific application cases are as follows. Zhu, Yuan and Ye [35] examined the multi-timescales of European carbon market with GRA method and the empirical mode decomposition. Tang, Zhu, Liu, Jia and Zheng [36] extended the TOPSIS with GRA to evaluate the urban sustainability. Tan, Chen and Wu [37] combined the AHP (analytical hierarchy process) with GRA to evaluate the environmentally conscious product design. Liu, Liu and $\mathrm{Hu}$ [38] evaluated the risks of mergers and acquisitions by GRA method under interval-valued intuitionistic fuzzy circumstance. However, the distance from the negative ideal point ignores the subjective factors such as the risk reference which influence the behavior of the decision makers in practice. Therefore, we extend the GRA method with cumulative prospect theory (CPT)Tversky and Kahneman [39] which improves the random dominance of prospect theory and shows the decision maker's performance under different environment.

With the conception of the fuzzy set (FS) by Zadeh [40], the extension of it such as the intuitionistic fuzzy set(IFS)[41] and hesitant fuzzy set (HFS)[42]. The HFS depict the complex decision-making environments in the real world using the memberships to describe each of the attribute. As the environment continues to be studied, the probabilistic hesitant fuzzy set (PHFS) was proposed by $\mathrm{Xu}$ and Zhou [43] to extend the original environment into a more general form. PHFS which solves the problem of DMs' choice of preference uses the probability to express different membership degrees and the HFS is the special form whose probabilities of the possible numbers are equal. Then the basic calculation formulas and related concepts has been proposed one after another such as the probabilistic hesitant fuzzy geometric operators [43] and distance measurement [44]. To avoid the different distance between probabilistic hesitant fuzzy elements (PHFEs), a new process of normalization was proposed by Li, Chen, Niu and Wang [45]. The PHFS is more consistent with behavior of DMs when making decisions, therefore, it is utilized in many 
fields. The TOPSIS method has been extended in Interval-Valued Pythagorean Fuzzy environment [46], Gaussian Interval Type-2 Fuzzy[47], Hesitant Fuzzy Linguistic environment [48] and so on. Thus, this paper is about to extended GRA method with CPT in probabilistic hesitant fuzzy circumstance.

The contributions to this article are as follows: (i) the CPT-GRA method expands the application of the classical GRA method for a better depict of the DMs' activity affected by psychological factors. (ii) the unknown weighting vectors of the attributes is also obtained by entropy weighted methods under probabilistic hesitant fuzzy environment. (iii) in the extended GRA which is based on CPT will be used in more practical situations.

The following is the main thread of the article. The basic knowledges are introduced in section 2. In section 3, an extended GRA method model of MAGDM is set up. In section 4, the newly proposed method is applied in the selection of green supplier. In section 5, the comparative analysis and the simulation analysis are made to show the practicability and validly. In section 6 , we draw a conclusion and prospect the future application.

\section{Preliminary Knowledge}

In this section, we excerpt some basics of probabilistic hesitant fuzzy set and novel GRA method based on cumulative prospect theory.

\subsection{Probabilistic Hesitant Fuzzy Sets}

Definition 1[43]. Set $y$ as a fixed set, a probabilistic hesitant fuzzy set (PHFS) on $y$ is represented as equation 1 :

$$
J=\left\{<y, k_{y}\left(t_{y}\right)>\mid y \in Y\right\}
$$

where the function $k_{y}\left(t_{y}\right)$ is the set of different numbers in $[0,1]$, denoting the possible membership degree of element, and the $t_{y}$ describes the probabilistic distribution. $k_{y} t_{(y)}$ is named the probabilistic hesitant fuzzy element (PHFE) and showed as $k(t)$, $0<k_{y}<1$.Probabilistic Hesitant Fuzzy Elements (PHFEs) can be presented by $k(t)=\left\{r_{l}\left(t_{l}\right) \mid l=1,2, \ldots, \# r\right\}$, where $t_{l}$ expresses the probability of the possible value $e_{l}$ and satisfies $\sum_{l=1}^{\# r} t_{l}=1$, where $\# r$ denotes the value of the membership. Then the proposition of a new normalization method[45] show us a different standard of the PHFEs to break the limitations 
of the original method.

Definition 2[45]. Set $k_{y}\left(t_{y}\right)=\left\{r_{y}\left(t_{y}\right)\right\}, k_{1}\left(t_{1}\right)=\left\{r_{a}(t)_{a}\right\}$ and $k_{2}\left(t_{2}\right)=\left\{r_{b}\left(t_{b}\right)\right\}$ be three PHFEs respectively. The specific steps of normalizing are as follows.

Step 1. Fix the first element of standardized PHFEs, where $k_{y}^{*}\left(t_{y}^{*}\right)$ represents the normalized PHFEs. If $t_{1}^{1}<t_{2}^{1}$, then $r_{1}^{1}\left(t_{1}^{1}\right)=r_{1}^{1}\left(t_{1}^{1}\right)$ and $r_{2}^{1}\left(t_{2}^{1}\right)=r_{2}^{1}\left(t_{1}^{1}\right)$, otherwise, $r_{1}^{1}\left(t_{1}^{1}\right)=r_{1}^{1}\left(t_{2}^{1}\right)$ and $r_{2}^{1}\left(t_{2}^{1}\right)=r_{2}^{1}\left(t_{2}^{1}\right)$

Step 2. Fix the second element of normalized PHFEs. If $t_{1}^{1}<t_{2}^{1}$ and $t_{2}^{1}-t_{1}^{1} \leq t_{1}^{2}$, then $r_{1}^{2}\left(t_{1}^{2}\right)=r_{1}^{2}\left(t_{2}^{1}-t_{1}^{1}\right) \quad$ and $\quad r_{2}^{2}\left(t_{2}^{2}\right)=r_{2}^{1}\left(t_{2}^{1}-t_{1}^{1}\right)$. If $\quad t_{1}^{1}<t_{2}^{1} \quad$ and $\quad t_{2}^{1}-t_{1}^{1}>t_{1}^{2} \quad, \quad$ then $r_{1}^{2}\left(t_{1}^{2}\right)=r_{1}^{2}\left(t_{1}^{2}\right)$ and $r_{2}^{2}\left(t_{2}^{2}\right)=r_{2}^{1}\left(t_{1}^{2}\right)$. If $t_{1}^{1} \geq t_{2}^{1}$ and $t_{1}^{1}-t_{2}^{1} \leq t_{2}^{2}$, then $r_{1}^{2}\left(t_{1}^{2}\right)=r_{1}^{1}\left(t_{1}^{1}-t_{2}^{1}\right)$ and $r_{2}^{2}\left(t_{2}^{2}\right)=r_{2}^{2}\left(t_{1}^{1}-t_{2}^{1}\right)$. If $t_{1}^{1} \geq t_{2}^{1} \quad$ and $\quad t_{1}^{1}-t_{2}^{1}>t_{2}^{2} \quad$ then $r_{1}^{2}\left(t_{1}^{2}\right)=r_{1}^{1}\left(t_{2}^{2}\right) \quad$ and $r_{2}^{2}\left(t_{2}^{2}\right)=r_{2}^{2}\left(t_{2}^{2}\right)$

Step 3. Fix the second element of normalized PHFEs. If $t_{1}^{1} \geq t_{2}^{1}, t_{1}^{1}-t_{2}^{1} \leq t_{2}^{2}$ and $t_{2}^{1} \leq t_{2}^{2}-t_{1}^{1}+t_{2}^{1}$, then $r_{1}^{3}\left(t_{1}^{3}\right)=r_{1}^{2}\left(t_{1}^{2}\right) \quad$ and $\quad r_{2}^{3}\left(t_{2}^{3}\right)=r_{2}^{2}\left(t_{1}^{2}\right)$. If $t_{1}^{1} \geq t_{2}^{1}, t_{1}^{1}-t_{2}^{1} \leq t_{2}^{2}$ and $t_{2}^{1}>t_{2}^{2}-t_{1}^{1}+t_{2}^{1}$, then $r_{1}^{3}\left(t_{1}^{3}\right)=r_{1}^{2}\left(t_{2}^{2}+t_{2}^{1}-t_{1}^{1}\right) \quad$ and $r_{2}^{3}\left(t_{2}^{3}\right)=r_{2}^{2}\left(t_{2}^{2}+t_{2}^{1}-t_{1}^{1}\right)$. If $t_{1}^{1} \geq t_{2}^{1}$, $t_{1}^{1}-t_{2}^{1}>t_{2}^{2} \quad$ and $t_{1}^{2} \geq t_{2}^{2}+t_{2}^{3}$, then $r_{1}^{3}\left(t_{1}^{3}\right)=r_{1}^{2}\left(t_{2}^{3}\right) \quad$ and $\quad r_{2}^{3}\left(t_{2}^{3}\right)=r_{2}^{3}\left(t_{2}^{3}\right) . \quad$ If $t_{1}^{1} \geq t_{2}^{1}$, $t_{1}^{1}-t_{2}^{1}>t_{2}^{2}$ and $t_{1}^{2}<t_{2}^{2}+t_{2}^{3}$, then $r_{1}^{3}\left(t_{1}^{3}\right)=r_{1}^{2}\left(t_{1}^{2}-t_{2}^{2}\right)$ and $r_{2}^{3}\left(t_{2}^{3}\right)=r_{2}^{3}\left(t_{1}^{2}-t_{2}^{2}\right)$. If $t_{1}^{1}<t_{2}^{1}$, $t_{2}^{1}-t_{1}^{1} \leq t_{1}^{2} \quad$ and $\quad t_{1}^{2}+t_{1}^{1} \leq t_{2}^{2}+t_{2}^{1} \quad, \quad$ then $\quad r_{1}^{3}\left(t_{1}^{3}\right)=r_{1}^{2}\left(t_{1}^{2}-t_{2}^{1}+t_{1}^{1}\right) \quad$ and $r_{2}^{3}\left(t_{2}^{3}\right)=r_{2}^{2}\left(t_{1}^{2}-t_{2}^{1}+t_{1}^{1}\right) \quad$. If $\quad t_{1}^{1}<t_{2}^{1} \quad, \quad t_{2}^{1}-t_{1}^{1}>t_{1}^{2} \quad$ and $\quad t_{2}^{1}+t_{1}^{2} \leq t_{1}^{3}+t_{1}^{1} \quad, \quad$ then $r_{1}^{3}\left(t_{1}^{3}\right)=r_{1}^{3}\left(t_{2}^{1}-t_{1}^{1}-t_{1}^{2}\right) \quad$ and $\quad r_{2}^{3}\left(t_{2}^{3}\right)=r_{2}^{1}\left(t_{2}^{1}-t_{1}^{1}-t_{1}^{2}\right)$. If $t_{1}^{1}<t_{2}^{1}, \quad t_{2}^{1}-t_{1}^{1}>t_{1}^{2} \quad$ and $t_{2}^{1}+t_{1}^{2}>t_{1}^{3}+t_{1}^{1}$, then $r_{1}^{3}\left(t_{1}^{3}\right)=r_{1}^{3}\left(t_{1}^{3}\right)$ and $r_{2}^{3}\left(t_{2}^{3}\right)=r_{2}^{1}\left(t_{1}^{3}\right)$

where $t_{1}^{1}+t_{1}^{2}+\cdots t_{1}^{\# r_{1}}=1$ and $t_{2}^{1}+t_{2}^{2}+\cdots t_{2}^{\# r_{2}}=1, \quad \# r_{1}=\# r_{2}$.

With the novel normalization method proposed, some calculation algorithms are introduced as follows. 
Definition 3[43]. For a normalized PHFE $k^{*}\left(t^{*}\right)=\left\{r^{*}\left(t^{*}\right)\right\}$, the score function is computed by Eq. (2)

$$
s\left(k^{*}\left(t^{*}\right)\right)=\sum_{l=1}^{\# r} r^{l *} f^{l *}
$$

where $\# r$ denotes the value of the diverse membership degrees, $r$ denotes the number of the possible values of the PHFE, $r^{l *}$ is the $l$ th largest elements, $t^{l *}$ is the $l$ th largest probability of the membership degree.

Definition 4[43]. For a PHFE $k^{*}\left(t^{*}\right)=\left\{r^{*}\left(t^{*}\right)\right\}$, the deviation degree of can be computed out by Eq. (3)

$$
d\left(k^{*}\left(t^{*}\right)\right)=\sum_{l=1}^{\# r} t^{l *}\left[r^{l *}-s\left(k^{*}\left(t^{*}\right)\right)\right]^{2}
$$

With the calculation of score and deviation, we can compare two PHFEs $k_{1}^{*}\left(t_{1}^{*}\right)$ and $k_{2}^{*}\left(t_{2}^{*}\right)$ by the following laws:

(1) $k_{1}^{*}\left(t_{1}^{*}\right)>k_{2}^{*}\left(t_{2}^{*}\right)$, if $s\left(k_{1}^{*}\left(t_{1}^{*}\right)\right)>s\left(k_{2}^{*}\left(t_{2}^{*}\right)\right)$

(2) $k_{1}^{*}\left(t_{1}^{*}\right)>k_{2}^{*}\left(t_{2}^{*}\right)$,if $s\left(k_{1}^{*}\left(t_{1}^{*}\right)\right)=s\left(k_{2}^{*}\left(t_{2}^{*}\right)\right)$ and $d\left(k_{1}^{*}\left(t_{1}^{*}\right)\right)<d\left(k_{2}^{*}\left(t_{2}^{*}\right)\right)$

(3) $k_{1}^{*}\left(t_{1}^{*}\right)=k_{2}^{*}\left(t_{2}^{*}\right)$,if $s\left(k_{1}^{*}\left(t_{1}^{*}\right)\right)=s\left(k_{2}^{*}\left(t_{2}^{*}\right)\right)$ and $d\left(k_{1}^{*}\left(t_{1}^{*}\right)\right)=d\left(k_{2}^{*}\left(t_{2}^{*}\right)\right)$

(4) $k_{1}^{*}\left(t_{1}^{*}\right)<k_{2}^{*}\left(t_{2}^{*}\right)$,if $s\left(k_{1}^{*}\left(t_{1}^{*}\right)\right)=s\left(k_{2}^{*}\left(t_{2}^{*}\right)\right)$ and $d\left(k_{1}^{*}\left(t_{1}^{*}\right)\right)>d\left(k_{2}^{*}\left(t_{2}^{*}\right)\right)$

Definition 5[49]. Set $k_{1}\left(t_{1}\right)$ and $k_{2}\left(t_{2}\right)$ be two PHFEs. And their normalized forms are $k_{1}^{*}\left(t_{1}^{*}\right)=\left\{r_{1}^{l}\left(t_{1}^{l}\right) \mid l=1,2 \cdots, \# r_{1}\right\} \quad$ and $\quad k_{2}^{*}\left(t_{2}^{*}\right)=\left\{r_{2}^{l}\left(t_{2}^{l}\right) \mid l=1,2 \cdots, \# r_{2}\right\} \quad, \quad$ where $\# r_{1}=\# r_{2}=\# r$ and $t_{1}^{l}=t_{2}^{l}=t^{l}$. The Lance distance between them is developed as Eq. (4)

$$
d\left(k_{1}^{*}\left(t_{1}^{*}\right), k_{2}^{*}\left(t_{2}^{*}\right)\right)=\frac{1}{\# r} \sum_{l=1}^{r} \frac{\left|r_{1}^{l *} t^{l *}-r_{2}^{l *} t^{l *}\right|}{r_{1}^{l *} t^{l *}+r_{2}^{l *} t^{l *}}
$$

$r^{l *}$ is the 1-th largest elements, $t^{l *}$ is the 1 -th largest probability of the membership.

Definition 6[45]. Let $k_{1}\left(t_{1}\right)$ and $k_{2}\left(t_{2}\right)$ be two PHFEs. And their normalized forms are $k_{1}^{*}\left(t_{1}^{*}\right)=\left\{r_{1}^{l}\left(t_{1}^{l}\right) \mid l=1,2 \cdots, \# r_{1}\right\} \quad$ and $\quad k_{2}^{*}\left(t_{2}^{*}\right)=\left\{r_{2}^{l}\left(t_{2}^{l}\right) \mid l=1,2 \cdots, \# r_{2}\right\} \quad$, where 
$\# r_{1}=\# r_{2}=\# r$ and $t_{1}^{l}=t_{2}^{l}=t^{l}$. The algorithms between them are as follows.

(1) $k_{1}^{*}\left(t_{1}^{*}\right) \oplus k_{2}^{*}\left(t_{2}^{*}\right)=\cup_{a=1, \cdots, \# r_{1}, b=1, \cdots, \# r_{2}}\left\{\left(e_{a}+e_{b}-e_{a} e_{b}\right)\left(f^{l}\right)\right\}$;

(2) $k_{1}^{*}\left(t_{1}^{*}\right) \otimes k_{2}^{*}\left(t_{2}^{*}\right)=\cup_{a=1, \cdots, \# r_{1}, b=1, \cdots, \# r_{2}}\left\{e_{a} e_{b}\left(f^{l}\right)\right\}$;

Definition 7. Let $D_{y}(c=1,2, \cdots, y)$ be an aggregation of PHFNs, and the algorithms of probabilistic hesitant fuzzy weighted averaging (PHFWA) operator is shown as Eq. (7)

$$
\begin{aligned}
& \operatorname{PHFWA}\left(k_{1}^{*}, k_{2}^{*}, \cdots, k_{y}^{*}\right)=\bigoplus_{c=1}^{y}\left(w_{c}^{*} q_{c}\right) \\
& =\underbrace{\cup}_{e_{1} \in D_{1}, e_{2} \in D_{2}, \cdots, e_{y} \in D_{y}}\left\{1-\prod_{c=1}^{y}\left(1-e_{c}\right)^{q_{c}}\left(f^{l}\right)\right\}
\end{aligned}
$$

where $q_{c}=\left(q_{1}, q_{2}, \cdots, q_{y}\right)$ is the weight vector of the $D_{y}$, and $\sum_{c=1}^{y} q_{c}=1, \quad q_{c} \in[0,1]$.

Definition 8. Let $D_{y}(c=1,2, \cdots, y)$ be a collection of PHFNs, and the equation of probabilistic hesitant fuzzy weighted geometric (PHFWG) operator is shown as follows.

$$
\begin{aligned}
P H F W G & =\left(k_{1}^{*}, k_{2}^{*}, \cdots, k_{y}^{*}\right)=\bigoplus_{c=1}^{y}\left(k_{c}^{*}\right)^{q_{c}} \\
& =\underbrace{}_{e_{1} \in D_{1}, e_{2} \in D_{2}, \cdots, e_{y} \in D_{y}}\left\{\prod_{c=1}^{y}\left(e_{c}\right)^{q_{c}}\left(f^{l}\right)\right\}
\end{aligned}
$$

2.4 An extended GRA method based on cumulative prospect theory under probabilistic hesitant fuzzy circumstance.

Let the MAGDM decision matrices be $K^{c}=\left[k_{m n}^{c}\left(t_{m n}\right)\right]_{i \times j}$, where $W=\left\{W_{1}, W_{2}, \cdots W_{i}\right\}$ stands for the alternatives, the attributes are represented as $P=\left\{P_{1}, P_{2}, \cdots P_{j}\right\}$, and $c=\left\{c_{1}, c_{2}, \cdots c_{y}\right\}$ is represented as the set of decision makers. Moreover, the weighting vector of attributes is unclear and the weight of decision makers is $D_{c}=\left(q_{1}, q_{2} \cdots q_{y}\right),(c=1,2, \cdots y)$, and meets the condition that $\sum_{c=1}^{y} q_{c}=1$. The specific steps of Probability Hesitant Fuzzy MAGDM using extended GRA method which is based CPT is as follows and its frame work are then shown in Figure 1.

The multiple attribute decision-making matrix is shown as follows: 


$$
K^{c}=\left[\begin{array}{ccccc}
k_{11}^{c} & \mathrm{~K} & k_{1 n}^{c} & \mathrm{~L} & k_{1 j}^{c} \\
\mathrm{M} & \mathrm{O} & \mathrm{M} & \mathrm{O} & \mathrm{M} \\
k_{m 1}^{c} & \mathrm{~L} & k_{m n}^{c} & \mathrm{~L} & k_{m j}^{c} \\
\mathrm{M} & \mathrm{O} & \mathrm{M} & \mathrm{O} & \mathrm{M} \\
k_{i 1}^{c} & \mathrm{~K} & k_{i n}^{c} & \mathrm{~L} & k_{i j}^{c}
\end{array}\right]_{i \times j} \quad ; m=1, \cdots, i, n=1, \cdots, j, c=1, \cdots, y
$$

The matrix $\left[o_{1}, \cdots, o_{j}\right]$ represents the weighting vector of attribute meeting the condition that $\sum_{n=1}^{j} o_{n}=1$ and $o_{n}$ is in $[0,1]$.

Step 1. Obtain the normalized decision matrices

In a real case, different metrics or different directions my appear in attributes which may cause the wrong result. Therefore, it is of necessity to normalize every element into the comparative one. The normalize steps are as follows.

Firstly, standardize for the directionality of attributes by using Eq. (9)

$$
\left\{\begin{array}{cl}
k_{m n}^{*}=\left\{r_{m n}\left(t_{m n}\right)\right\} & \text { if the attribute is the positive attribute } \\
k_{m n}^{*}=\left\{\left(1-r_{m n}\right)\left(t_{m n}\right)\right\} & \text { if the attribute is the negative attribute }
\end{array}\right.
$$

where $w_{k g}^{*}$ represents the value of normalized decision matrix.

Then normalize the decision matrices using the steps in Definition 2.

Step 2. Integrate the normalized group decision matrices into a decision matrix $\bar{K}=\left[\bar{k}_{m n}\left(\bar{t}_{m n}\right)\right]_{i \times j}$ by using the PHFWA which calculated by Eq. (10)

$$
\begin{aligned}
\operatorname{PHFWA}\left(k_{1}^{*}, k_{2}^{*}, \cdots, k_{y}^{*}\right)=\bigoplus_{c=1}^{y}\left(k_{c}^{*} q_{c}\right) \\
=\underbrace{}_{r_{1}^{*} \in k_{1}^{*}, r_{2}^{*} \in k_{2}^{*}, \cdots, r_{c}^{*} \in k_{c}^{*}}\left\{1-\prod_{c=1}^{y}\left(1-r_{c}^{*}\right)^{q_{c}}\left(t^{l}\right)\right\}
\end{aligned}
$$

Step 3. Figure out the score function of the integrated decision matrix.

$$
s\left(\bar{k}_{m n}\left(\bar{t}_{m n}\right)\right)=\sum_{l=1}^{\# r} \bar{r}_{m n}^{l} \bar{f}_{m n}^{l}
$$

Step 4. Compute out the weights among attributes

The weighting vector of each attribute is completely unknown in this condition, so it's either given directly or computed. Owing to the fact that in probabilistic hesitant fuzzy environment every prospect decision element should have their corresponding different attributes weight. We use the 
entropy weighting method and its calculating law is as follows.

$$
\bar{o}_{n}=\frac{1-\bar{h}_{n}}{\sum_{n=1}^{j}\left(1-\bar{h}_{n}\right)} \quad n=1,2, \cdots, j
$$

where $\bar{h}_{n}$ denotes the entropy of the $\mathrm{n}$-th attributes and calculate it by Eq. (13)

$$
\bar{h}_{n}=-\frac{1}{\ln i} \sum_{m=1}^{i}\left[\frac{s\left(\bar{k}_{m n}\left(\bar{t}_{m n}\right)\right)}{\sum_{m=1}^{i} s\left(\bar{k}_{m n}\left(\bar{t}_{m n}\right)\right)} \ln \left(\frac{s\left(\bar{k}_{m n}\left(\bar{t}_{m n}\right)\right)}{\sum_{m=1}^{i} s\left(\bar{k}_{m n}\left(\bar{t}_{m n}\right)\right)}\right)\right]
$$

Step5. Definite the positive ideal solution (PIS) $g^{+}$and negative ideal solution (NIS) $g^{-}$ separately.

$$
g^{+}=\left\{\bar{k}_{1}^{+}, \cdots \bar{k}_{j}^{+}\right\}=\left\{\max _{m}\left(\bar{k}_{m n}\right)\right\}
$$

where $\bar{k}_{n}^{+}\left(\bar{t}_{n}^{+}\right)=\left\{\bar{r}_{n}^{+}\left(\bar{t}_{n}^{+}\right)\right\}=\max _{m} s\left(\bar{k}_{m n}\left(\bar{t}_{m n}\right)\right)$

$$
g^{-}=\left\{\bar{k}_{1}^{-}, \cdots \bar{k}_{j}^{-}\right\}=\left\{\min _{m} \bar{k}_{m n}\right\}
$$

where $\bar{k}_{n}^{-}\left(\bar{t}_{n}^{-}\right)=\left\{{\overline{r_{n}}}^{-}\left(\bar{t}_{n}^{-}\right)\right\}=\min _{m} s\left(\bar{k}_{m n}\left(\bar{t}_{m n}\right)\right)$

Step 6. Calculate the distances of between the positive and negative ideal point using Eq.

$$
\left\{\begin{array}{l}
d\left(\bar{k}_{m n}\left(\bar{t}_{m n}\right), \bar{k}_{n}^{+}\left(\bar{t}^{+}\right)\right)=\frac{1}{\# r} \sum_{l=1}^{r} \frac{\left|\bar{r}_{m n}^{l} \bar{t}^{l}-\bar{r}_{n}^{l+} \bar{t}^{l+}\right|}{\bar{r}_{m n}^{l} \bar{t}^{l}+\bar{r}_{n}^{l+} \bar{t}^{l+}} \\
d\left(\bar{k}_{m n}\left(\bar{t}_{m n}\right), \bar{k}_{n}^{-}\left(\bar{t}^{-}\right)\right)=\frac{1}{\# r} \sum_{l=1}^{r} \frac{\left|\bar{r}_{m n}^{l} \bar{t}^{l}-\bar{r}_{n}^{l-} \bar{t}^{l-}\right|}{\bar{r}_{m n}^{l} \bar{t}^{l}+\bar{r}_{n}^{l-} \bar{t}^{l-}} \quad n=1, \cdots, j
\end{array}\right.
$$

where $\# r$ denotes the value of the possible membership of every element.

Step 7. Figure out the grey relational coefficient of each alternative

The grey relational coefficient between feasible scheme and PIS

$$
\xi_{m n}^{+}=\frac{\min _{m} \min _{n} d\left(\bar{k}_{m n}\left(\bar{t}_{m n}\right), \bar{k}_{n}^{+}\left(\bar{t}^{+}\right)\right)+\rho \max _{m} \max _{n} d\left(\bar{k}_{m n}\left(\bar{t}_{m n}\right), \bar{k}_{n}^{+}\left(\bar{t}^{+}\right)\right)}{d\left(\bar{k}_{m n}\left(\bar{t}_{m n}\right), \bar{k}_{n}^{+}\left(\bar{t}^{+}\right)\right)+\rho \max _{m} \max _{n} d\left(\bar{k}_{m n}\left(\bar{t}_{m n}\right), \bar{k}_{n}^{+}\left(\bar{t}^{+}\right)\right)}
$$

The grey relational coefficient between feasible scheme and NIS

$$
\xi_{m n}^{-}=\frac{\min _{m} \min _{n} d\left(\bar{k}_{m n}\left(\bar{t}_{m n}\right), \bar{k}_{n}^{-}\left(\bar{t}^{-}\right)\right)+\rho \max _{m} \max _{n} d\left(\bar{k}_{m n}\left(\bar{t}_{m n}\right), \bar{k}_{n}^{-}\left(\bar{t}^{-}\right)\right)}{d\left(\bar{k}_{m n}\left(\bar{t}_{m n}\right), \bar{k}_{n}^{-}\left(\bar{t}^{-}\right)\right)+\rho \max _{m} \max _{n} d\left(\bar{k}_{m n}\left(\bar{t}_{m n}\right), \bar{k}_{n}^{-}\left(\bar{t}^{-}\right)\right)}
$$

where the identification coefficient $\rho=0.5$. 
Step 8. Figure out the positive and negative value function $v_{m n}^{+(-)}$.

$$
\left\{\begin{array}{cc}
v_{m n}^{+}=\left(\left(1-\xi_{m n}^{-}\right)^{\alpha}\right. & \text { when DMs are faced with gains } \\
v_{m n}^{-}=-\theta\left(-\left(\xi_{m n}^{+}-1\right)\right)^{\beta} & \text { when DMs are faced with losses }
\end{array}\right.
$$

where $\alpha$ and $\beta$ are the parameters and $\theta$ is the parameter deriving from the experiment of Tversky and Kahneman [39]( $\lambda=2.25, \alpha=0.88, \beta=0.88)$ which express the sensitivity of the risk aversion.

Step 9. Obtain the transformed probability weights for the gains and losses $\pi_{m n}^{+(-)}$which can be calculated by the following equations:

$$
\left\{\begin{array}{l}
\pi_{m n}^{+}=F^{+}\left(\sum_{e=1}^{j} o_{n}\right)-F^{+}\left(\sum_{e=n+1}^{j} o_{n}\right) \text { when DMs are faced with gains } \\
\pi_{m n}^{-}=F^{-}\left(\sum_{e=1}^{n} o_{n}\right)-F^{-}\left(\sum_{e=1}^{n-1} o_{n}\right) \quad \text { when DMs are faced with losses }
\end{array}\right.
$$

where the nonlinear functions of gains $F^{+}\left(o_{n}\right)$ and losses $F^{-}\left(o_{n}\right)$ separately and can be represented as the following rules:

$$
\left\{\begin{array}{l}
F^{+}\left(o_{n}\right)=\frac{o_{n}^{\gamma}}{\left(o_{n}^{\gamma}+\left(1-o_{n}\right)^{\gamma}\right)^{\frac{1}{\gamma}}} \quad \text { when DMs are faced with gains } \\
F^{-}\left(o_{n}\right)=\frac{o_{n}^{\sigma}}{\left(o_{n}^{\sigma}+\left(1-o_{n}\right)^{\sigma}\right)^{\frac{1}{\sigma}}} \quad \text { when DMs are faced with losses }
\end{array}\right.
$$

where $\gamma$ and $\sigma$ represent the attitude of decision maker towards gains and losses and these parameters are from the experiment of Tversky and Kahneman [39] ( $\gamma=0.61$ and $\sigma=0.69$ ).

Step 10. Obtain the prospect matrix $V_{m}$

$$
V_{m}=\sum_{n=1}^{j} \pi_{m n}^{+}\left(o_{n}\right) \cdot v_{m n}^{+}+\sum_{n=1}^{j} \pi_{m n}^{-}\left(o_{n}\right) \cdot v_{m n}^{-}
$$

where $V_{m}$ denotes the optimal comprehensive prospect value of alternative $W_{m}$.

Step 11. Rank the optimal comprehensive prospect value of each scheme in a descending order. 
Step 3: obtain the

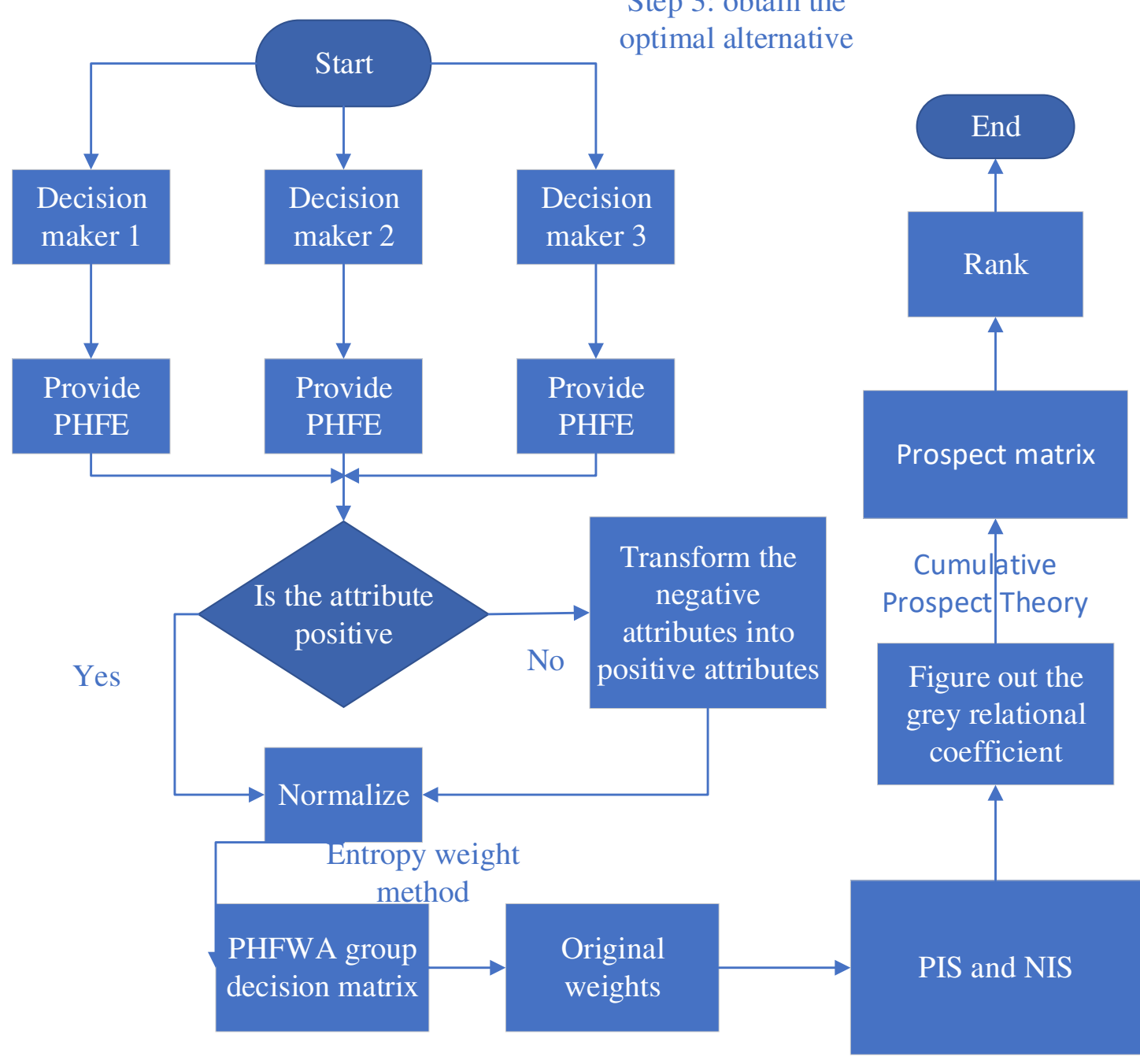

Step 1: normalize and integrate matrix

Step 2:determine the PIS and NIS

Figure 1. the Framework of the Probability Hesitant Fuzzy MAGDM using extended GRA

\section{3. the numerical example}

The concept of green supplier selection, also known as the environmentally conscious supply chain, is proposed to develop the product production processes such as the material acquisition, processing, packaging, warehousing, transportation and use to scrap, so that they have the least impact on the environment (negative effect) and the highest resource efficiency. In recent years, with the concept of environmental protection deeply rooted in people's hearts, the green supplier selection is taken seriously by more and more enterprises. Normally, six evaluation principles to evaluate the green supplier: $P_{1}$ cost factor; $P_{2}$ product factor; $P_{3}$ green supply chain management; $P_{4}$ green imagination of enterprise; $P_{5}$ service capability; $P_{6}$ enterprise 
qualification. Considering an enterprise need to select the most optimal supplier from five different green supplier selection $W=\left\{W_{1}, W_{2}, \cdots W_{i}\right\}$ by evaluate them in six attributes $P=\left\{P_{1}, P_{2}, \cdots P_{j}\right\}$. In order to select the best alternative, there are three experts $c=\left\{c_{1}, c_{2}, \cdots c_{y}\right\}$, and each of them have more than five years of experience. Moreover, the weighting vector of attributes is unclear and the weight of decision makers is $D_{c}=(0.3,0.4,0.3)$. The following Table1-3 is the decisionmaking matrices under probabilistic hesitant fuzzy circumstance of this case study which is given by three experts.

Table 1. Decision matrix $K_{1}$ given by the first expert

\begin{tabular}{cccc}
\hline Alternative & $\mathbf{P}_{\mathbf{1}}$ & $\mathbf{P}_{\mathbf{2}}$ & $\mathbf{P}_{\mathbf{3}}$ \\
\hline $\mathbf{W}_{\mathbf{1}}$ & $\{0.8(0.7), 0.3(0.3)\}$ & $\{0.4(1)\}$ & $\{0.3(0.5), 0.1(0.2), 0.2(0.3)\}$ \\
\hline $\mathbf{W}_{\mathbf{2}}$ & $\{0.3(0.2), 0.2(0.5), 0.1(0.3)\}$ & $\{0.2(0.2), 0.3(0.5), 0.1(0.3)\}$ & $\{0.1(0.2), 0.2(0.5), 0.4(0.3)\}$ \\
\hline $\mathbf{W}_{\mathbf{3}}$ & $\{0.1(0.2), 0.2(0.5), 0.3(0.3)\}$ & $\{0.5(0.5), 0.1(0.5)\}$ & $\{0.4(0.5), 0.2(0.5)\}$ \\
\hline $\mathbf{W}_{\mathbf{4}}$ & $\{0.6(0.5), 0.5(0.5)\}$ & $\{0.7(0.7), 0.5(0.3)\}$ & $\{0.7(1)\}$ \\
\hline $\mathbf{W}_{\mathbf{5}}$ & $\{0.3(1)\}$ & $\{0.3(0.5), 0.2(0.5)\}$ & $\{0.3(0.5), 0.2(0.2), 0.1(0.3)\}$ \\
\hline Alternative & $\mathbf{P}_{\mathbf{4}}$ & $\mathbf{P}_{\mathbf{5}}$ & $\mathbf{\mathbf { P } _ { \mathbf { 6 } }}$ \\
\hline $\mathbf{W}_{\mathbf{1}}$ & $\{0.2(0.2), 0.3(0.3), 0.4(0.5)\}$ & $\{0.4(0.2), 0.3(0.3), 0.1(0.5)\}$ & $\{0.2(0.7), 0.3(0.3)\}$ \\
\hline $\mathbf{W}_{\mathbf{2}}$ & $\{0.3(0.7), 0.2(0.3)\}$ & $\{0.2(0.5), 0.3(0.5)\}$ & $\{0.3(0.5), 0.2(0.5)\}$ \\
\hline $\mathbf{W}_{\mathbf{3}}$ & $\{0.2(0.5), 0.3(0.5)\}$ & $\{0.1(0.7), 0.3(0.3\}$ & $\{0.4(1)\}$ \\
\hline $\mathbf{W}_{\mathbf{4}}$ & $\{0.6(0.7), 0.7(0.3)\}$ & $\{0.5(0.2), 0.6(0.8)\}$ & $\{0.6(0.2), 0.7(0.8)\}$ \\
\hline & $\{0.3(0.2), 0.4(0.3), 0.2(0.5)\}$ & $\{0.2(0.5), 0.3(0.2), 0.1(0.3)\}$ & $\{0.3(0.2), 0.2(0.5), 0.4(0.3)\}$ \\
\hline
\end{tabular}

Table 2. Decision matrix $K_{2}$ given by the second expert 


\begin{tabular}{cccc}
\hline Alternative & $\mathbf{P}_{\mathbf{1}}$ & $\mathbf{P}_{\mathbf{2}}$ & $\mathbf{P}_{\mathbf{3}}$ \\
\hline $\mathbf{W}_{\mathbf{1}}$ & $\{0.4(0.2), 0.2(0.5), 0.3(0.3)\}$ & $\{0.2(0.2), 0.4(0.5), 0.1(0.3)\}$ & $\{0.2(1)\}$ \\
\hline $\mathbf{W}_{\mathbf{2}}$ & $\{0.5(0.5), 0.2(0.5)\}$ & $\{0.2(0.2), 0.1(0.5), 0.4(0.3)\}$ & $\{0.5(0.2), 0.3(0.3), 0.2(0.5)\}$ \\
\hline $\mathbf{W}_{\mathbf{3}}$ & $\{0.3(1)\}$ & $\{0.3(0.7), 0.2(0.3)\}$ & $\{0.3(0.7), 0.2(0.3)\}$ \\
\hline $\mathbf{W}_{\mathbf{4}}$ & $\{0.6(0.7), 0.7(0.3)\}$ & $\{0.7(0.5), 0.5(0.5)\}$ & $\{0.9(0.2), 0.4(0.5), 0.7(0.3)\}$ \\
\hline $\mathbf{W}_{\mathbf{5}}$ & $\{0.3(0.5), 0.2(0.5)\}$ & $\{0.1(1)\}$ & $\{0.1(0.5), 0.2(0.5)\}$ \\
\hline Alternative & $\mathbf{P}_{\mathbf{4}}$ & $\mathbf{P}_{\mathbf{5}}$ & $\mathbf{P}_{\mathbf{6}}$ \\
\hline $\mathbf{W}_{\mathbf{1}}$ & $\{0.2(0.5), 0.3(0.5)\}$ & $\{0.1(0.2), 0.3(0.5), 0.2(0.3)\}$ & $\{0.2(0.5), 0.3(0.5)\}$ \\
\hline $\mathbf{W}_{\mathbf{2}}$ & $\{0.1(0.2), 0.5(0.5), 0.3(0.3)\}$ & $\{0.3(0.5), 0.4(0.5)\}$ & $\{0.1(0.2), 0.3(0.5), 0.4(0.3)\}$ \\
\hline $\mathbf{W}_{\mathbf{3}}$ & $\{0.3(0.7), 0.4(0.3)\}$ & $\{0.4(1)\}$ & $\{0.3(0.2), 0.1(0.3), 0.2(0.5)\}$ \\
\hline $\mathbf{W}_{\mathbf{4}}$ & $\{0.8(0.2), 0.5(0.3), 0.6(0.5)\}$ & $\{0.7(0.2), 0.8(0.8)\}$ & $\{0.8(0.2), 0.5(0.8)\}$ \\
\hline $\mathbf{5}$ & $\{0.3(1)\}$ & $\{0.2(0.2), 0.4(0.5), 0.1(0.3)\}$ & $\{0.2(1)\}$ \\
\hline
\end{tabular}

Table 3. Decision matrix $K_{3}$ given by the third expert

\begin{tabular}{cccc}
\hline Alternative & $\mathbf{P}_{\mathbf{1}}$ & $\mathbf{P}_{\mathbf{2}}$ & $\mathbf{P}_{\mathbf{3}}$ \\
\hline $\mathbf{W}_{\mathbf{1}}$ & $\{0.2(0.2), 0.3(0.5), 0.4(0.3)\}$ & $\{0.3(0.5), 0.2(0.5)\}$ & $\{0.1(0.2), 0.3(0.3), 0.4(0.5)\}$ \\
\hline $\mathbf{W}_{\mathbf{2}}$ & $\{0.2(1)\}$ & $\{0.2(0.2), 0.3(0.5), 0.4(0.3)\}$ & $\{0.2(0.7), 0.1(0.3)\}$ \\
\hline $\mathbf{W}_{\mathbf{3}}$ & $\{0.3(0.7), 0.2(0.3)\}$ & $\{0.3(0.7), 0.4(0.3)\}$ & $\{0.4(0.5), 0.2(0.5)\}$ \\
\hline $\mathbf{W}_{\mathbf{4}}$ & $\{0.7(0.2), 0.8(0.5), 0.9(0.3)\}$ & $\{0.5(0.2), 0.4(0.5), 0.6(0.3)\}$ & $\{0.8(0.2), 0.6(0.5), 0.3(0.3)\}$ \\
\hline $\mathbf{W}_{\mathbf{5}}$ & $\{0.3(0.5), 0.4(0.5)\}$ & $\{0.3(0.2), 0.1(0.5), 0.2(0.3)\}$ & $\{0.3(0.7), 0.2(0.3)\}$ \\
\hline Alternative & $\mathbf{P}_{\mathbf{4}}$ & $\mathbf{P}_{\mathbf{5}}$ & $\mathbf{P}_{\mathbf{6}}$ \\
\hline $\mathbf{W}_{\mathbf{1}}$ & $\{0.2(0.2), 0.1(0.5), 0.4(0.3)\}$ & $\{0.3(0.2), 0.4(0.8)\}$ & $\{0.1(0.2), 0.4(0.8)\}$ \\
\hline
\end{tabular}




\begin{tabular}{cccc}
\hline $\mathbf{W}_{\mathbf{2}}$ & $\{0.3(0.5), 0.4(0.5)\}$ & $\{0.1(0.5), 0.2(0.5)\}$ & $\{0.2(0.2), 0.1(0.3), 0.3(0.5)\}$ \\
\hline $\mathbf{W}_{\mathbf{3}}$ & $\{0.1(0.7), 0.2(0.3)\}$ & $\{0.2(1)\}$ & $\{0.2(0.5), 0.1(0.5)\}$ \\
\hline $\mathbf{W}_{\mathbf{4}}$ & $\{0.6(0.5), 0.8(0.5)\}$ & $\{0.5(0.2), 0.9(0.5), 0.8(0.3)\}$ & $\{0.8(0.2), 0.3(0.3), 0.7(0.5)\}$ \\
\hline $\mathbf{W}_{\mathbf{5}}$ & $\{0.2(1)\}$ & $\{0.2(0.2), 0.3(0.3), 0.1(0.5)\}$ & $\{0.3(1)\}$ \\
\hline
\end{tabular}

Step 1. Standardize the decision matrices and its results are shown in Table 4 to 6.

Table 4. Normalized decision matrix $K_{1}$ given by the first expert

\begin{tabular}{|c|c|c|}
\hline Alternative & $\mathbf{P}_{1}$ & $\mathbf{P}_{2}$ \\
\hline $\mathbf{W}_{1}$ & $\{0.2(0.2), 0.2(0.3), 0.2(0.2), 0.3(0.3)\}$ & $\{0.4(0.2), 0.4(0.3), 0.4(0.2), 0.4(0.3)\}$ \\
\hline $\mathbf{W}_{2}$ & $\{0.3(0.2), 0.2(0.3), 0.2(0.2), 0.1(0.3)\}$ & $\{0.2(0.2), 0.3(0.3), 0.3(0.2), 0.1(0.3)\}$ \\
\hline $\mathbf{W}_{3}$ & $\{0.1(0.2), 0.2(0.3), 0.2(0.2), 0.3(0.3)\}$ & $\{0.5(0.2), 0.5(0.3), 0.1(0.2), 0.1(0.3)\}$ \\
\hline $\mathbf{W}_{4}$ & $\{0.6(0.2), 0.6(0.3), 0.5(0.2), 0.5(0.3)\}$ & $\{0.7(0.2), 0.7(0.3), 0.7(0.2), 0.5(0.3)\}$ \\
\hline $\mathbf{W}_{5}$ & $\{0.3(0.2), 0.3(0.3), 0.3(0.2), 0.3(0.3)\}$ & $\{0.3(0.2), 0.3(0.3), 0.2(0.2), 0.2(0.3)\}$ \\
\hline Alternative & $\mathbf{P}_{3}$ & $\mathbf{P}_{4}$ \\
\hline $\mathbf{W}_{1}$ & $\{0.3(0.2), 0.3(0.3), 0.1(0.2), 0.2(0.3)\}$ & $\{0.2(0.2), 0.3(0.3), 0.4(0.2), 0.4(0.3)\}$ \\
\hline $\mathbf{W}_{2}$ & $\{0.1(0.2), 0.2(0.3), 0.2(0.2), 0.4(0.3)\}$ & $\{0.3(0.2), 0.3(0.3), 0.3(0.2), 0.2(0.3)\}$ \\
\hline $\mathbf{W}_{\mathbf{3}}$ & $\{0.4(0.2), 0.4(0.3), 0.2(0.2), 0.2(0.3)\}$ & $\{0.2(0.2), 0.2(0.3), 0.3(0.2), 0.3(0.3)\}$ \\
\hline $\mathbf{W}_{4}$ & $\{0.7(0.2), 0.7(0.3), 0.7(0.2), 0.7(0.3)\}$ & $\{0.6(0.2), 0.6(0.3), 0.6(0.2), 0.7(0.3)\}$ \\
\hline $\mathbf{W}_{5}$ & $\{0.3(0.2), 0.3(0.3), 0.2(0.2), 0.1(0.3)\}$ & $\{0.3(0.2), 0.4(0.3), 0.2(0.2), 0.2(0.3)\}$ \\
\hline Alternative & $\mathbf{P}_{5}$ & $\mathbf{P}_{6}$ \\
\hline $\mathbf{W}_{1}$ & $\{0.4(0.2), 0.3(0.3), 0.1(0.2), 0.1(0.3)\}$ & $\{0.2(0.2), 0.2(0.3), 0.2(0.2), 0.3(0.3)\}$ \\
\hline $\mathbf{W}_{2}$ & $\{0.2(0.2), 0.2(0.3), 0.3(0.2), 0.3(0.3)\}$ & $\{0.3(0.2), 0.3(0.3), 0.2(0.2), 0.2(0.3)\}$ \\
\hline
\end{tabular}




\begin{tabular}{ccc}
\hline $\mathbf{W}_{\mathbf{3}}$ & $\{0.1(0.2), 0.1(0.3), 0.1(0.2), 0.3(0.3)\}$ & $\{0.4(0.2), 0.4(0.3), 0.4(0.2), 0.4(0.3)\}$ \\
\hline $\mathbf{W}_{\mathbf{4}}$ & $\{0.5(0.2), 0.6(0.3), 0.6(0.2), 0.6(0.3)\}$ & $\{0.6(0.2), 0.7(0.3), 0.7(0.2), 0.7(0.3)\}$ \\
\hline $\mathbf{W}_{\mathbf{5}}$ & $\{0.2(0.2), 0.2(0.3), 0.3(0.2), 0.1(0.3)\}$ & $\{0.3(0.2), 0.2(0.3), 0.2(0.2), 0.4(0.3)\}$ \\
\hline
\end{tabular}

Table 5. Normalized decision matrix $K_{2}$ given by the second expert

\begin{tabular}{|c|c|c|}
\hline Alternative & $\mathbf{P}_{1}$ & $\mathbf{P}_{2}$ \\
\hline $\mathbf{W}_{1}$ & $\{0.4(0.2), 0.2(0.3), 0.2(0.2), 0.3(0.3)\}$ & $\{0.2(0.2), 0.4(0.3), 0.4(0.2), 0.1(0.3)\}$ \\
\hline $\mathbf{W}_{2}$ & $\{0.5(0.2), 0.5(0.3), 0.2(0.2), 0.2(0.3)\}$ & $\{0.2(0.2), 0.1(0.3), 0.1(0.2), 0.4(0.3)\}$ \\
\hline $\mathbf{W}_{3}$ & $\{0.3(0.2), 0.3(0.3), 0.3(0.2), 0.3(0.3)\}$ & $\{0.3(0.2), 0.3(0.3), 0.3(0.2), 0.2(0.3)\}$ \\
\hline $\mathbf{W}_{4}$ & $\{0.6(0.2), 0.6(0.3), 0.6(0.2), 0.7(0.3)\}$ & $\{0.7(0.2), 0.7(0.3), 0.5(0.2), 0.5(0.3)\}$ \\
\hline $\mathbf{W}_{5}$ & $\{0.3(0.2), 0.3(0.3), 0.2(0.2), 0.2(0.3)\}$ & $\{0.1(0.2), 0.1(0.3), 0.1(0.2), 0.1(0.3)\}$ \\
\hline Alternative & $\mathbf{P}_{3}$ & $\mathbf{P}_{4}$ \\
\hline $\mathbf{W}_{1}$ & $\{0.2(0.2), 0.2(0.3), 0.2(0.2), 0.2(0.3)\}$ & $\{0.2(0.2), 0.2(0.3), 0.3(0.2), 0.3(0.3)\}$ \\
\hline $\mathbf{W}_{2}$ & $\{0.5(0.2), 0.3(0.3), 0.2(0.2), 0.2(0.3)\}$ & $\{0.1(0.2), 0.5(0.3), 0.5(0.2), 0.3(0.3)\}$ \\
\hline $\mathbf{W}_{3}$ & $\{0.3(0.2), 0.3(0.3), 0.3(0.2), 0.2(0.3)\}$ & $\{0.3(0.2), 0.3(0.3), 0.3(0.2), 0.4(0.3)\}$ \\
\hline $\mathbf{W}_{4}$ & $\{0.9(0.2), 0.4(0.3), 0.4(0.2), 0.7(0.3)\}$ & $\{0.8(0.2), 0.5(0.3), 0.6(0.2), 0.6(0.3)\}$ \\
\hline $\mathbf{W}_{5}$ & $\{0.1(0.2), 0.1(0.3), 0.2(0.2), 0.2(0.3)\}$ & $\{0.3(0.2), 0.3(0.3), 0.3(0.2), 0.3(0.3)\}$ \\
\hline Alternative & $\mathbf{P}_{5}$ & $\mathbf{P}_{6}$ \\
\hline $\mathbf{W}_{1}$ & $\{0.1(0.2), 0.3(0.3), 0.3(0.2), 0.2(0.3)\}$ & $\{0.2(0.2), 0.2(0.3), 0.3(0.2), 0.3(0.3)\}$ \\
\hline $\mathbf{W}_{2}$ & $\{0.3(0.2), 0.3(0.3), 0.4(0.2), 0.4(0.3)\}$ & $\{0.1(0.2), 0.3(0.3), 0.3(0.2), 0.4(0.3)\}$ \\
\hline $\mathbf{W}_{\mathbf{3}}$ & $\{0.4(0.2), 0.4(0.3), 0.4(0.2), 0.4(0.3)\}$ & $\{0.3(0.2), 0.1(0.3), 0.2(0.2), 0.2(0.3)\}$ \\
\hline $\mathbf{W}_{4}$ & $\{0.7(0.2), 0.8(0.3), 0.8(0.2), 0.8(0.3)\}$ & $\{0.8(0.2), 0.5(0.3), 0.5(0.2), 0.5(0.3)\}$ \\
\hline
\end{tabular}


$\mathbf{W}_{5}$

$\{0.2(0.2), 0.4(0.3), 0.4(0.2), 0.1(0.3)\}$

$\{0.2(0.2), 0.2(0.3), 0.2(0.2), 0.2(0.3)\}$

Table 6. Normalized decision matrix $K_{3}$ given by the third expert

\begin{tabular}{|c|c|c|}
\hline Alternative & $\mathbf{P}_{1}$ & $\mathbf{P}_{2}$ \\
\hline $\mathbf{W}_{1}$ & $\{0.2(0.2), 0.3(0.3), 0.3(0.2), 0.4(0.3)\}$ & $\{0.3(0.2), 0.3(0.3), 0.2(0.2), 0.2(0.3)\}$ \\
\hline $\mathbf{W}_{2}$ & $\{0.2(0.2), 0.2(0.3), 0.2(0.2), 0.2(0.3)\}$ & $\{0.2(0.2), 0.3(0.3), 0.3(0.2), 0.4(0.3)\}$ \\
\hline $\mathbf{W}_{3}$ & $\{0.3(0.2), 0.3(0.3), 0.3(0.2), 0.2(0.3)\}$ & $\{0.3(0.2), 0.3(0.3), 0.3(0.2), 0.4(0.3)\}$ \\
\hline $\mathbf{W}_{4}$ & $\{0.7(0.2), 0.8(0.3), 0.8(0.2), 0.9(0.3)\}$ & $\{0.5(0.2), 0.4(0.3), 0.4(0.2), 0.6(0.3)\}$ \\
\hline $\mathbf{W}_{5}$ & $\{0.3(0.2), 0.3(0.3), 0.4(0.2), 0.4(0.3)\}$ & $\{0.3(0.2), 0.1(0.3), 0.1(0.2), 0.2(0.3)\}$ \\
\hline Alternative & $\mathbf{P}_{3}$ & $\mathbf{P}_{4}$ \\
\hline $\mathbf{W}_{1}$ & $\{0.1(0.2), 0.3(0.3), 0.4(0.2), 0.4(0.3)\}$ & $\{0.2(0.2), 0.1(0.3), 0.1(0.2), 0.4(0.3)\}$ \\
\hline $\mathbf{W}_{2}$ & $\{0.2(0.2), 0.2(0.3), 0.2(0.2), 0.1(0.3)\}$ & $\{0.3(0.2), 0.3(0.3), 0.4(0.2), 0.4(0.3)\}$ \\
\hline $\mathbf{W}_{3}$ & $\{0.4(0.2), 0.4(0.3), 0.2(0.2), 0.2(0.3)\}$ & $\{0.1(0.2), 0.1(0.3), 0.1(0.2), 0.2(0.3)\}$ \\
\hline $\mathbf{W}_{4}$ & $\{0.8(0.2), 0.6(0.3), 0.6(0.2), 0.3(0.3)\}$ & $\{0.6(0.2), 0.6(0.3), 0.8(0.2), 0.8(0.3)\}$ \\
\hline $\mathbf{W}_{5}$ & $\{0.3(0.2), 0.3(0.3), 0.3(0.2), 0.2(0.3)\}$ & $\{0.2(0.2), 0.2(0.3), 0.2(0.2), 0.2(0.3)\}$ \\
\hline Alternative & $\mathbf{P}_{5}$ & $\mathbf{P}_{6}$ \\
\hline $\mathbf{W}_{1}$ & $\{0.3(0.2), 0.4(0.3), 0.4(0.2), 0.4(0.3)\}$ & $\{0.1(0.2), 0.4(0.3), 0.4(0.2), 0.4(0.3)\}$ \\
\hline $\mathbf{W}_{2}$ & $\{0.1(0.2), 0.1(0.3), 0.2(0.2), 0.2(0.3)\}$ & $\{0.2(0.2), 0.1(0.3), 0.3(0.2), 0.3(0.3)\}$ \\
\hline $\mathbf{W}_{3}$ & $\{0.2(0.2), 0.2(0.3), 0.2(0.2), 0.2(0.3)\}$ & $\{0.2(0.2), 0.2(0.3), 0.1(0.2), 0.1(0.3)\}$ \\
\hline $\mathbf{W}_{4}$ & $\{0.5(0.2), 0.9(0.3), 0.9(0.2), 0.8(0.3)\}$ & $\{0.8(0.2), 0.3(0.3), 0.7(0.2), 0.7(0.3)\}$ \\
\hline $\mathbf{W}_{5}$ & $\{0.2(0.2), 0.3(0.3), 0.1(0.2), 0.1(0.3)\}$ & $\{0.3(0.2), 0.3(0.3), 0.3(0.2), 0.3(0.3)\}$ \\
\hline
\end{tabular}

Step 2. Integrate the standardized decision matrices into group decision matrix using the Eq. 
(10), and the overall decision matrix is shown in Table 7.

Table 7. the overall decision matrix

\begin{tabular}{|c|c|c|}
\hline Alternative & $\mathbf{P}_{1}$ & $\mathbf{P}_{2}$ \\
\hline $\mathbf{W}_{1}$ & $\{0.287(0.2), 0.231(0.3), 0.231(0.2), 0.332(0.3)\}$ & $\{0.295(0.2), 0.372(0.3), 0.346(0.2), 0.231(0.3)\}$ \\
\hline $\mathbf{W}_{2}$ & $\{0.363(0.2), 0.337(0.3), 0.200(0.2), 0.171(0.3)\}$ & $\{0.200(0.2), 0.226(0.3), 0.226(0.2), 0.322(0.3)\}$ \\
\hline $\mathbf{W}_{3}$ & $\{0.245(0.2), 0.271(0.3), 0.271(0.2), 0.271(0.3)\}$ & $\{0.367(0.2), 0.367(0.3), 0.245(0.2), 0.240(0.3)\}$ \\
\hline $\mathbf{W}_{4}$ & $\{0.633(0.2), 0.675(0.3), 0.653(0.2), 0.748(0.3)\}$ & $\{0.650(0.2), 0.631(0.3), 0.547(0.2), 0.532(0.3)\}$ \\
\hline $\mathbf{W}_{5}$ & $\{0.300(0.2), 0.300(0.3), 0.295(0.2), 0.295(0.3)\}$ & $\{0.226(0.2), 0.165(0.3), 0.131(0.2), 0.161(0.3)\}$ \\
\hline Alternative & $\mathbf{P}_{3}$ & $\mathbf{P}_{4}$ \\
\hline $\mathbf{W}_{1}$ & $\{0.204(0.2), 0.262(0.3), 0.240(0.2), 0.266(0.3)\}$ & $\{0.200(0.2), 0.204(0.3), 0.279(0.2), 0.362(0.3)\}$ \\
\hline $\mathbf{W}_{2}$ & $\{0.313(0.2), 0.242(0.3), 0.200(0.2), 0.240(0.3)\}$ & $\{0.226(0.2), 0.388(0.3), 0.416(0.2), 0.304(0.3)\}$ \\
\hline $\mathbf{W}_{3}$ & $\{0.362(0.2), 0.362(0.3), 0.242(0.2), 0.242(0.3)\}$ & $\{0.214(0.2), 0.214(0.3), 0.245(0.2), 0.315(0.3)\}$ \\
\hline $\mathbf{W}_{4}$ & $\{0.829(0.2), 0.568(0.3), 0.568(0.2), 0.613(0.3)\}$ & $\{0.697(0.2), 0.563(0.3), 0.675(0.2), 0.702(0.3)\}$ \\
\hline $\mathbf{W}_{5}$ & $\{0.226(0.2), 0.226(0.3), 0.231(0.2), 0.171(0.3)\}$ & $\{0.271(0.2), 0.304(0.3), 0.242(0.2), 0.242(0.3)\}$ \\
\hline Alternative & $\mathbf{P}_{5}$ & $\mathbf{P}_{6}$ \\
\hline $\mathbf{W}_{1}$ & $\{0.261(0.2), 0.332(0.3), 0.279(0.2), 0.240(0.3)\}$ & $\{0.171(0.2), 0.266(0.3), 0.304(0.2), 0.332(0.3)\}$ \\
\hline $\mathbf{W}_{2}$ & $\{0.214(0.2), 0.214(0.3), 0.315(0.2), 0.315(0.3)\}$ & $\{0.194(0.2), 0.245(0.3), 0.271(0.2), 0.315(0.3)\}$ \\
\hline $\mathbf{W}_{3}$ & $\{0.261(0.2), 0.261(0.3), 0.261(0.2), 0.315(0.3)\}$ & $\{0.304(0.2), 0.231(0.3), 0.240(0.2), 0.240(0.3)\}$ \\
\hline $\mathbf{W}_{4}$ & $\{0.592(0.2), 0.800(0.3), 0.800(0.2), 0.754(0.3)\}$ & $\{0.754(0.2), 0.525(0.3), 0.632(0.2), 0.632(0.3)\}$ \\
\hline $\mathbf{W}_{5}$ & $\{0.200(0.2), 0.315(0.3), 0.290(0.2), 0.100(0.3)\}$ & $\{0.262(0.2), 0.231(0.3), 0.231(0.2), 0.295(0.3)\}$ \\
\hline
\end{tabular}

Step 3. Figure out the score function of the integrated decision matrix. 
Table 8 The score of the group decision matrix

\begin{tabular}{ccccccc}
\hline & $\mathbf{P}_{\mathbf{1}}$ & $\mathbf{P}_{\mathbf{2}}$ & $\mathbf{P}_{\mathbf{3}}$ & $\mathbf{P}_{\mathbf{4}}$ & $\mathbf{P}_{\mathbf{5}}$ & $\mathbf{P}_{\mathbf{6}}$ \\
\hline $\mathbf{W}_{\mathbf{1}}$ & 0.273 & 0.309 & 0.247 & 0.266 & 0.279 & 0.274 \\
$\mathbf{W}_{\mathbf{2}}$ & 0.265 & 0.250 & 0.247 & 0.336 & 0.265 & 0.261 \\
$\mathbf{W}_{\mathbf{3}}$ & 0.266 & 0.305 & 0.289 & 0.251 & 0.277 & 0.250 \\
$\mathbf{W}_{\mathbf{4}}$ & 0.684 & 0.588 & 0.634 & 0.654 & 0.745 & 0.624 \\
$\mathbf{W}_{\mathbf{5}}$ & 0.297 & 0.169 & 0.211 & 0.266 & 0.223 & 0.257 \\
\hline
\end{tabular}

Step 4. Compute out the weights among attributes using the entropy and its result is as follows. $o_{n}=\{0.160,0.156,0.175,0.143,0.220,0.145\}$.

Step 5. Determine the positive ideal solution (PIS) $g^{+}$and negative ideal solution (NIS) $g^{-}$ in table 9 and 10 .

Table 9 The positive ideal solution

\begin{tabular}{|c|c|c|c|c|c|}
\hline $\mathbf{P}_{1}$ & $\mathbf{P}_{2}$ & $\mathbf{P}_{3}$ & $\mathbf{P}_{4}$ & $\mathbf{P}_{5}$ & $\mathbf{P}_{6}$ \\
\hline$\{0.633(0.2)$ & $\{0.650(0.2)$ & $\{0.829(0.2)$ & $\{0.697(0.2)$ & $\{0.592(0.2)$ & $\{0.754(0.2)$ \\
\hline $0.675(0.3)$ & $0.631(0.3)$ & $0.568(0.3)$ & $0.563(0.3)$ & $0.800(0.3)$ & $0.525(0.3)$ \\
\hline $0.653(0.2)$ & $0.547(0.2)$ & $0.568(0.2)$ & $0.675(0.2)$ & $0.800(0.2)$ & $0.632(0.2)$ \\
\hline $0.748(0.3)\}$ & $0.532(0.3)\}$ & $0.613(0.3)\}$ & $0.702(0.3)\}$ & $0.754(0.3)\}$ & $0.632(0.3)\}$ \\
\hline
\end{tabular}

Table 10 The negative ideal solution

$\begin{array}{llllll}\mathbf{P}_{1} & \mathbf{P}_{2} & \mathbf{P}_{3} & \mathbf{P}_{4} & \mathbf{P}_{5} & \mathbf{P}_{6}\end{array}$




\begin{tabular}{llllll}
\hline$\{0.363(0.2)$, & $\{0.226(0.2)$, & $\{0.226(0.2)$, & $\{0.214(0.2)$, & $\{0.200(0.2)$, & $\{0.304(0.2)$, \\
$0.337(0.3)$, & $0.165(0.3)$, & $0.226(0.3)$, & $0.214(0.3)$, & $0.315(0.3)$, & $0.231(0.3)$, \\
$0.200(0.2)$, & $0.131(0.2)$, & $0.231(0.2)$, & $0.245(0.2)$, & $0.290(0.2)$, & $0.240(0.2)$, \\
$0.171(0.3)\}$ & $0.161(0.3)\}$ & $0.171(0.3)\}$ & $0.315(0.3)\}$ & $0.100(0.3)\}$ & $0.240(0.3)\}$ \\
\hline
\end{tabular}

Step 6. Calculate the distance between the positive and negative ideal point separately, and the results are shown in Table 11 and 12.

Table 11 The distance between the PIS

\begin{tabular}{ccccccc}
\hline & $\mathbf{P}_{\mathbf{1}}$ & $\mathbf{P}_{\mathbf{2}}$ & $\mathbf{P}_{\mathbf{3}}$ & $\mathbf{P}_{\mathbf{4}}$ & $\mathbf{P}_{\mathbf{5}}$ & $\mathbf{P}_{\mathbf{6}}$ \\
\hline $\mathbf{W}_{\mathbf{1}}$ & 0.432 & 0.315 & 0.447 & 0.440 & 0.451 & 0.409 \\
$\mathbf{W}_{\mathbf{2}}$ & 0.452 & 0.419 & 0.443 & 0.334 & 0.474 & 0.425 \\
$\mathbf{W}_{\mathbf{3}}$ & 0.437 & 0.326 & 0.383 & 0.457 & 0.454 & 0.429 \\
$\mathbf{W}_{\mathbf{4}}$ & 0.000 & 0.000 & 0.000 & 0.000 & 0.000 & 0.000 \\
$\mathbf{W}_{\mathbf{5}}$ & 0.389 & 0.554 & 0.498 & 0.427 & 0.556 & 0.425 \\
\hline
\end{tabular}

Table 12 The distance between the NIS

\begin{tabular}{ccccccc}
\hline & $\mathbf{P}_{\mathbf{1}}$ & $\mathbf{P}_{\mathbf{2}}$ & $\mathbf{P}_{\mathbf{3}}$ & $\mathbf{P}_{\mathbf{4}}$ & $\mathbf{P}_{\mathbf{5}}$ & $\mathbf{P}_{\mathbf{6}}$ \\
\hline $\mathbf{W}_{\mathbf{1}}$ & 0.040 & 0.289 & 0.090 & 0.049 & 0.142 & 0.100 \\
$\mathbf{W}_{\mathbf{2}}$ & 0.000 & 0.174 & 0.103 & 0.141 & 0.113 & 0.082 \\
$\mathbf{W}_{\mathbf{3}}$ & 0.074 & 0.279 & 0.141 & 0.000 & 0.138 & 0.000 \\
$\mathbf{W}_{\mathbf{4}}$ & 0.452 & 0.554 & 0.498 & 0.457 & 0.556 & 0.429 \\
$\mathbf{W}_{\mathbf{5}}$ & 0.107 & 0.000 & 0.000 & 0.047 & 0.000 & 0.049 \\
\hline
\end{tabular}

Step 7. Figure out the grey relational coefficient of each alternative and the grey relational 
coefficient between PIS and NIS are shown in Table 13 and 14.

Table 13 The grey relational coefficient between PIS

\begin{tabular}{ccccccc}
\hline & $\mathbf{P}_{\mathbf{1}}$ & $\mathbf{P}_{\mathbf{2}}$ & $\mathbf{P}_{\mathbf{3}}$ & $\mathbf{P}_{\mathbf{4}}$ & $\mathbf{P}_{\mathbf{5}}$ & $\mathbf{P}_{\mathbf{6}}$ \\
\hline $\mathbf{W}_{\mathbf{1}}$ & 0.391 & 0.469 & 0.383 & 0.387 & 0.381 & 0.404 \\
$\mathbf{W}_{\mathbf{2}}$ & 0.381 & 0.399 & 0.385 & 0.454 & 0.370 & 0.395 \\
$\mathbf{W}_{\mathbf{3}}$ & 0.389 & 0.460 & 0.420 & 0.378 & 0.380 & 0.393 \\
$\mathbf{W}_{\mathbf{4}}$ & 1.000 & 1.000 & 1.000 & 1.000 & 1.000 & 1.000 \\
$\mathbf{W}_{\mathbf{5}}$ & 0.417 & 0.334 & 0.358 & 0.394 & 0.333 & 0.395 \\
\hline
\end{tabular}

Table 14 The grey relational coefficient between NIS

\begin{tabular}{ccccccc}
\hline & $\mathbf{P}_{\mathbf{1}}$ & $\mathbf{P}_{\mathbf{2}}$ & $\mathbf{P}_{\mathbf{3}}$ & $\mathbf{P}_{\mathbf{4}}$ & $\mathbf{P}_{\mathbf{5}}$ & $\mathbf{P}_{\mathbf{6}}$ \\
\hline $\mathbf{W}_{\mathbf{1}}$ & 0.875 & 0.490 & 0.755 & 0.851 & 0.662 & 0.736 \\
$\mathbf{W}_{\mathbf{2}}$ & 1.000 & 0.615 & 0.730 & 0.664 & 0.711 & 0.772 \\
$\mathbf{W}_{\mathbf{3}}$ & 0.791 & 0.499 & 0.664 & 1.000 & 0.668 & 1.000 \\
$\mathbf{W}_{\mathbf{4}}$ & 0.381 & 0.334 & 0.358 & 0.378 & 0.333 & 0.393 \\
$\mathbf{W}_{\mathbf{5}}$ & 0.723 & 1.000 & 1.000 & 0.856 & 1.000 & 0.849 \\
\hline
\end{tabular}

Step 8. Figure out the positive and negative value function $v_{m n}^{+(-)}$and show its result in Table 15 and 16.

Table 15 The positive prospect value

\begin{tabular}{ccccccc}
\hline & $\mathbf{P}_{\mathbf{1}}$ & $\mathbf{P}_{\mathbf{2}}$ & $\mathbf{P}_{\mathbf{3}}$ & $\mathbf{P}_{\mathbf{4}}$ & $\mathbf{P}_{\mathbf{5}}$ & $\mathbf{P}_{\mathbf{6}}$ \\
\hline $\mathbf{W}_{\mathbf{1}}$ & 0.161 & 0.553 & 0.290 & 0.187 & 0.385 & 0.310 \\
$\mathbf{W}_{\mathbf{2}}$ & 0.000 & 0.432 & 0.316 & 0.383 & 0.335 & 0.272 \\
$\mathbf{W}_{\mathbf{3}}$ & 0.252 & 0.544 & 0.383 & 0.000 & 0.379 & 0.000 \\
\hline
\end{tabular}




\begin{tabular}{lllllll}
\hline $\mathbf{W}_{4}$ & 0.656 & 0.699 & 0.677 & 0.658 & 0.700 & 0.644 \\
$\mathbf{W}_{5}$ & 0.323 & 0.000 & 0.000 & 0.182 & 0.000 & 0.190 \\
\hline
\end{tabular}

Table 16 The negative prospect value

\begin{tabular}{ccccccc}
\hline & $\mathbf{P}_{\mathbf{1}}$ & $\mathbf{P}_{\mathbf{2}}$ & $\mathbf{P}_{\mathbf{3}}$ & $\mathbf{P}_{\mathbf{4}}$ & $\mathbf{P}_{\mathbf{5}}$ & $\mathbf{P}_{\mathbf{6}}$ \\
\hline $\mathbf{W}_{\mathbf{1}}$ & -1.454 & -1.289 & -1.470 & -1.462 & -1.474 & -1.426 \\
$\mathbf{W}_{\mathbf{2}}$ & -1.475 & -1.438 & -1.466 & -1.321 & -1.499 & -1.445 \\
$\mathbf{W}_{\mathbf{3}}$ & -1.459 & -1.308 & -1.393 & -1.481 & -1.478 & -1.450 \\
$\mathbf{W}_{\mathbf{4}}$ & 0.000 & 0.000 & 0.000 & 0.000 & 0.000 & 0.000 \\
$\mathbf{W}_{\mathbf{5}}$ & -1.400 & -1.574 & -1.523 & -1.447 & -1.575 & -1.446 \\
\hline
\end{tabular}

Step 9. Obtain the transformed probability weights for the gains and losses $\pi_{m n}^{+(-)}$.

$$
\begin{aligned}
& \pi^{+}=\{0.356,0.476,0.575,0.647,0.776,1.000\} \\
& \pi^{-}=\{0.225,0.113,0.111,0.093,0.181,0.278\}
\end{aligned}
$$

Step 10. Obtain the prospect matrix $V_{m}$ of each alternative.

$$
V_{m}=\{-0.217,-0.284,-0.574,2.569,-1.059\}
$$

Step 11. Rank

$$
W_{4}>W_{1}>W_{2}>W_{3}>W_{5}
$$

\section{Comparative Analysis}

In this section, we will first apply the same data into different methods to testify the effectiveness of the newly proposed method. Then in order to further prove the applicability and feasibility of the method, sensitive and simulation analysis are also applied in this article.

\subsection{Comparative Analysis with other methods}

we will use kinds of methods whose classical TODIM (PHF-TODIM) method[50], PHFWA 
operator[43], PHFWG operator[43], probabilistic hesitant fuzzy TOPSIS (PHF-TOPSIS) method[51] and the novel TODIM method proposed by Tian, Niu, Ma and Xu [52] to compare with GRA based on CPT method. To make a better comparison, we use the same data from the Table 1 to Table 3 and the attributes weight vector which is obtained from Step 4 is as follows:

$o_{n}=\{0.160,0.156,0.175,0.143,0.220,0.145\}$

\subsubsection{Comparison with PHF-TODIM method}

The classical TODIM method under probabilistic hesitant fuzzy condition proposed by Zhang, Du and Tian [50] is one of the most commonly used evaluation methods to solve the selection issues. Thus, we compare it with the developed GRA method. The following is the detailed process of this method.

Firstly, use the data of Table 7 as the group decision making matrix, and the weighting vector of attributes is $o_{n}=\{0.160,0.156,0.175,0.143,0.220,0.145\}$, and then the relative weight can be calculated by Eq. (38)

$$
o_{n}^{*}=\frac{o_{n}}{\hat{o}}
$$

where $\hat{e}$ denotes the maximum value of the attributes' weight.

And then compute out the dominance degree of the alternative $W_{m}$ over the other alternatives are shown as Eq. (24)

$$
\tau\left(W_{m}\right)=\tau\left(W_{m}, W_{m^{\prime}}\right)=\sum_{n=1}^{n} \xi_{m m^{\prime} n}\left(W_{m}, W_{m^{\prime}}\right)
$$

In Eq. (25), the preference index number is computed. 


$$
\xi_{m m^{\prime} n}\left(W_{m}, W_{m^{\prime}}\right) \begin{cases}\sqrt{\frac{o_{n}^{*} \times d\left(k_{m n}, k_{m^{\prime} n}\right)}{\sum_{n=1}^{j} o_{n}^{*}}} & \text { if } \mathrm{s}\left(k_{m n}\right) \geq s\left(k_{m^{\prime} n}\right) \\ -\theta \sqrt{\frac{\sum_{n=1}^{j} o_{n}^{*} \times d\left(k_{m n}, k_{m^{\prime} n}\right)}{o_{n}^{*}}} & \text { if } \mathrm{s}\left(k_{m n}\right)<s\left(k_{m^{\prime} n}\right)\end{cases}
$$

Finally, the overall dominance degree of the alternative can be obtained by Eq. (26).

$$
\vartheta\left(W_{n}\right)=\frac{\tau\left(W_{n}\right)-\min _{m}\left(\tau\left(W_{m}\right)\right)}{\max _{m}\left(\tau\left(W_{m}\right)\right)-\min _{m}\left(\tau\left(W_{m}\right)\right)} m=1,2, \ldots, i
$$

Table 17. The outcomes about PHF-TODIM method

Appellation Sign The results

The

relative weights

$$
o_{n}^{*}=\frac{o_{n}}{\hat{o}}
$$

$$
B_{n}=(0.724,0.707,0.796,0.651,1.000,0.660)
$$

The overall

degree

$$
\vartheta\left(W_{n}\right)
$$$$
\vartheta\left(W_{1}\right)=0.258, \vartheta\left(W_{2}\right)=0.164, \vartheta\left(W_{3}\right)=0.142, \vartheta\left(W_{4}\right)=1.000, \vartheta\left(W_{5}\right)=0.000
$$

The result

$$
W_{4}>W_{1}>W_{2}>W_{3}>W_{5}
$$

\subsubsection{Comparison with Probabilistic Hesitant Fuzzy Weighting Aggregation Operators}

Aggregation operator is used as the most intuitive measurement data to solve the MAGDM problems. The result of probabilistic hesitant fuzzy aggregation operators is shown in Table 18 and 19.

Table 18. The outcome about PHFWA operator 


\begin{tabular}{rll}
\hline $\mathbf{W}_{\mathbf{1}}$ & $\{0.240(0.2), 0.284(0.3), 0.280(0.2), 0.290(0.3)\}$ & 0.2762 \\
\hline $\mathbf{W}_{\mathbf{2}}$ & $\{0.255(0.2), 0.273(0.3), 0.275(0.2), 0.280(0.3)\}$ & 0.2719 \\
\hline $\mathbf{W}_{\mathbf{3}}$ & $\{0.295(0.2), 0.288(0.3), 0.252(0.2), 0.266(0.3)\}$ & 0.2757 \\
\hline $\mathbf{W}_{\mathbf{4}}$ & $\{0.701(0.2), 0.655(0.3), 0.667(0.2), 0.678(0.3)\}$ & 0.6734 \\
\hline $\mathbf{W}_{\mathbf{5}}$ & $\{0.245(0.2), 0.262(0.3), 0.242(0.2), 0.205(0.3)\}$ & 0.2375 \\
\hline The result & $W_{4}>W_{1}>W_{3}>W_{2}>W_{5}$ & \\
\hline
\end{tabular}

Table 19. The outcome about PHFWG operator

\begin{tabular}{ccc}
\hline Alternative & Overall values & Score \\
\hline $\mathbf{W}_{\mathbf{1}}$ & $\{0.234(0.2), 0.276(0.3), 0.276(0.2), 0.284(0.3)\}$ & 0.27019 \\
\hline $\mathbf{W}_{\mathbf{2}}$ & $\{0.245(0.2), 0.263(0.3), 0.262(0.2), 0.272(0.3)\}$ & 0.26194 \\
\hline $\mathbf{W}_{\mathbf{3}}$ & $\{0.287(0.2), 0.280(0.3), 0.251(0.2), 0.262(0.3)\}$ & 0.27017 \\
\hline $\mathbf{W}_{\mathbf{4}}$ & $\{0.683(0.2), 0.632(0.3), 0.648(0.2), 0.664(0.3)\}$ & 0.65496 \\
\hline $\mathbf{W}_{\mathbf{5}}$ & $\{0.241(0.2), 0.254(0.3), 0.233(0.2), 0.187(0.3)\}$ & 0.22705 \\
\hline The result & $W_{4}>W_{1}>W_{3}>W_{2}>W_{5}$ & \\
\hline
\end{tabular}

\subsubsection{Comparison with PHF-TOPSIS method}

The TOPSIS method which is similar to the GRA method in theory and principles under probabilistic hesitant fuzzy circumstance proposed by Wu, Liu, Wang and Zhang [51] can be applied to testify the effectiveness of the developed method.

Firstly, used the value of Table 7 as the standardized value of decision matrix and the weighting vector of attributes is $o_{n}=\{0.160,0.156,0.175,0.143,0.220,0.145\}$, and based on the score 
function, figure out the negative and positive ideal point. Meanwhile, calculate the distance between the negative and positive ideal point $D_{m}^{-}$and $D_{m}^{+}$separately. Then obtained the relative closeness coefficients of each alternative by Eq. (27)

$$
F^{*}=\frac{D_{m}^{-}}{D_{m}^{-}+D_{m}^{+}}
$$

where $D_{m}^{+}$and $D_{m}^{-}$can be computed by the distance measurement introduced in Definition 5 .

Table 20. The outcomes about PHF-TOPSIS method

\begin{tabular}{lcc}
\hline Appellation & Sign & The results \\
\hline $\begin{array}{l}\text { The distance } \\
D_{m}^{+}\end{array}$ & $D_{m}^{+}=(2.494,2.547,2.485,0.000,2.848)$ \\
The relative & $D_{m}^{-}=(0.709,0.612,0.631,2.945,0.203)$ \\
closeness & $F_{m}^{*}$ & $F_{1}^{*}=0.221, F_{2}^{*}=0.194, F_{3}^{*}=0.203, F_{4}^{*}=1.000, F_{5}^{*}=0.066$ \\
coefficients & \\
The result & $W_{4}>W_{1}>W_{2}>W_{3}>W_{5}$ \\
\hline
\end{tabular}

\subsection{Comprehension Comparison}

According to Table 16, the results of different methods are kind of different and the optimal alternative is $W_{4}$. The reason why there are a little difference are as follows. The classical TODIM method neglect to distinguish the negative and positive attributes but focus on MADM in real number. Besides, it regards the initial weight vector of the attributes as the ultima weight vector. While the PHFWA and the PHFWG operator focus on the entirety and unit separately, and the TOPSIS method pays more attention to the disparity of the negative and positive ideal point. Results calculated using the same data are compared by different methods to prove the practicability of the new method preliminarily. 
Table 21. The sequence from different methods

\begin{tabular}{ccc}
\hline Method & The sequence & The best alternative \\
\hline PHF-TODIM[50] & $W_{4}>W_{1}>W_{2}>W_{3}>W_{5}$ & $W_{4}$ \\
PHFWA operator[43] & $W_{4}>W_{1}>W_{3}>W_{2}>W_{5}$ & $W_{4}$ \\
PHFWG operator[43] & $W_{4}>W_{1}>W_{3}>W_{2}>W_{5}$ & $W_{4}$ \\
PHF-TOPSIS[51] & $W_{4}>W_{1}>W_{3}>W_{2}>W_{5}$ & $W_{4}$ \\
PHF-CPT-GRA & $W_{4}>W_{1}>W_{2}>W_{3}>W_{5}$ & $W_{4}$ \\
\hline
\end{tabular}

\section{Sensitive and Simulative Analysis}

In order to better prove the stability and effectiveness of the new method, we apply the sensitive analysis and the simulation analysis. According to the results of these two analyses, the superiority of the developed method has been proved.

\subsubsection{Sensitive Analysis of the Extended GRA and the GRA method with Probabilistic}

\section{Hesitant Fuzzy Information Based on the Distinguishing Parameter $\rho$.}

To further illustrate the superiority of the extended GRA method based on CPT under probabilistic hesitant fuzzy circumstance, this section we calculate the extend GRA method and classical GRA with probabilistic hesitant fuzzy information by changing the distinguishing parameter $\rho$.

The distinguishing parameter $\rho$ is the only changeable parameter in both extended GRA and classical GRA method. By changing the value of $\rho(0<\rho \leq 1)$, and the numerical results of the extended GRA method and classical GRA method can be shown in Table 22 and Table 23 and the normalized data of the Table 22 and 23 to draw the relevancy degree changes curve with MATLAB software can be shown in Figure 2.

Table 22. ranking order with different value of $\rho$ with Extended GRA method in probabilistic hesitant fuzzy environment

\begin{tabular}{lllllll}
\hline$\rho$ & $\mathbf{W}_{1}$ & $\mathbf{W}_{2}$ & $\mathbf{W}_{3}$ & $\mathbf{W}_{4}$ & $\mathbf{W}_{5}$ & Rank \\
\hline
\end{tabular}




\begin{tabular}{llllllll}
\hline 0.1 & 0.523 & 0.407 & -0.388 & 3.480 & -0.946 & $W_{4}>W_{1}>W_{2}>W_{3}>W_{5}$ \\
0.2 & 0.136 & 0.055 & -0.509 & 3.192 & -1.090 & $W_{4}>W_{1}>W_{2}>W_{3}>W_{5}$ \\
0.3 & -0.053 & -0.126 & -0.558 & 2.951 & -1.112 & $W_{4}>W_{1}>W_{2}>W_{3}>W_{5}$ \\
0.4 & -0.157 & -0.226 & -0.574 & 2.746 & -1.093 & $W_{4}>W_{1}>W_{2}>W_{3}>W_{5}$ \\
0.5 & -0.217 & -0.284 & -0.574 & 2.569 & -1.059 & $W_{4}>W_{1}>W_{2}>W_{3}>W_{5}$ \\
0.6 & -0.253 & -0.319 & -0.566 & 2.415 & -1.020 & $W_{4}>W_{1}>W_{2}>W_{3}>W_{5}$ \\
0.7 & -0.274 & -0.338 & -0.553 & 2.280 & -0.980 & $W_{4}>W_{1}>W_{2}>W_{3}>W_{5}$ \\
0.8 & -0.287 & -0.349 & -0.538 & 2.160 & -0.941 & $W_{4}>W_{1}>W_{2}>W_{3}>W_{5}$ \\
0.9 & -0.293 & -0.353 & -0.523 & 2.052 & -0.904 & $W_{4}>W_{1}>W_{2}>W_{3}>W_{5}$ \\
1 & -0.295 & -0.354 & -0.507 & 1.956 & -0.869 & $W_{4}>W_{1}>W_{2}>W_{3}>W_{5}$ \\
\hline
\end{tabular}

Table 23. ranking order with different value of $\rho$ with Classical GRA method

probabilistic hesitant fuzzy environment

\begin{tabular}{rrrrrcc}
\hline$\rho$ & $\mathbf{W}_{\mathbf{1}}$ & $\mathbf{W}_{\mathbf{2}}$ & $\mathbf{W}_{\mathbf{3}}$ & $\mathbf{W}_{\mathbf{4}}$ & $\mathbf{W}_{\mathbf{5}}$ & Rank \\
\hline 0.1 & 0.702 & 0.657 & 0.768 & 1.786 & 0.353 & $W_{4}>W_{3}>W_{1}>W_{2}>W_{5}$ \\
0.2 & 0.689 & 0.661 & 0.753 & 1.730 & 0.411 & $W_{4}>W_{3}>W_{1}>W_{2}>W_{5}$ \\
0.3 & 0.703 & 0.680 & 0.760 & 1.683 & 0.466 & $W_{4}>W_{3}>W_{1}>W_{2}>W_{5}$ \\
0.4 & 0.724 & 0.703 & 0.775 & 1.643 & 0.514 & $W_{4}>W_{3}>W_{1}>W_{2}>W_{5}$ \\
0.5 & 0.746 & 0.726 & 0.792 & 1.608 & 0.577 & $W_{4}>W_{3}>W_{1}>W_{2}>W_{5}$ \\
0.6 & 0.768 & 0.748 & 0.809 & 1.578 & 0.595 & $W_{4}>W_{3}>W_{1}>W_{2}>W_{5}$ \\
0.7 & 0.788 & 0.769 & 0.825 & 1.552 & 0.628 & $W_{4}>W_{3}>W_{1}>W_{2}>W_{5}$ \\
0.8 & 0.807 & 0.789 & 0.841 & 1.529 & 0.658 & $W_{4}>W_{3}>W_{1}>W_{2}>W_{5}$ \\
0.9 & 0.824 & 0.806 & 0.855 & 1.508 & 0.685 & $W_{4}>W_{3}>W_{1}>W_{2}>W_{5}$ \\
\hline
\end{tabular}




$\begin{array}{lllllll}1 & 0.840 & 0.823 & 0.869 & 1.489 & 0.708 & W_{4}>W_{3}>W_{1}>W_{2}>W_{5}\end{array}$
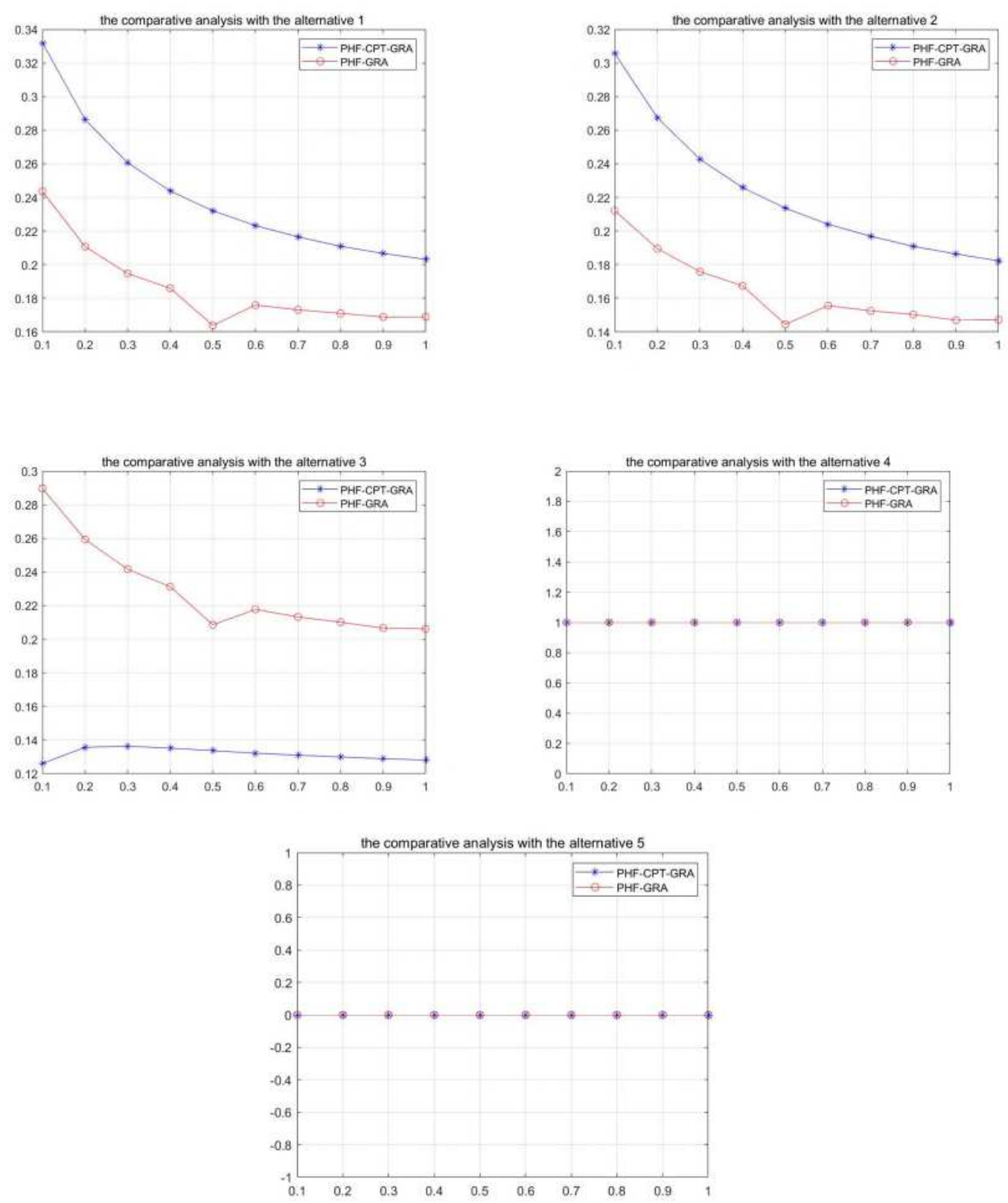

Figure 2. sensitive analysis with probabilistic hesitant fuzzy information $0<\rho \leq 1, \lambda=2.25, \alpha=0.88, \beta=0.88$

To better represent trends in the graph, we further standardized the results of Table 22 ad Table 23. According to the Figure 2, when $\rho$ varies, the curve of the extended GRA is more smooth than the one obtained by the classical GRA. In the comparison of alternative 1 and 2 , the fluctuation range of the extended GRA method is bigger than the one obtained by the GRA method, while the 
fluctuation range of extended GRA method in alternative 3 is smaller than the one gained by classical GRA method. Moreover, no matter what the parameters are, the final ranking is all equal.

\subsubsection{Sensitive Analysis of the Extended GRA with Probabilistic Hesitant Fuzzy} Information and Hesitant Fuzzy Information Based on the Distinguishing Parameter $\rho$.

In this section, we use the extended GRA method with the probabilistic hesitant fuzzy information and fuzzy hesitant fuzzy information separately to find the sensitivity of the extended method to parameters in different environment. The numerical result of the probabilistic hesitant fuzzy environment and fuzzy hesitant fuzzy environment are shown in Table 22 and Table24. Besides, the standardized relevancy degree changes curve with MATLAB software can be shown in Figure 3.

Table 24. ranking order with different value of $\rho$ with extended GRA method in hesitant fuzzy information

\begin{tabular}{rrrrrrc}
\hline$\rho$ & $\mathbf{W}_{\mathbf{1}}$ & $\mathbf{W}_{\mathbf{2}}$ & $\mathbf{W}_{\mathbf{3}}$ & $\mathbf{W}_{\mathbf{4}}$ & $\mathbf{W}_{\mathbf{5}}$ & Rank \\
\hline 0.1 & 1.265 & 1.147 & -1.148 & 3.471 & 0.201 & $W_{4}>W_{1}>W_{2}>W_{5}>W_{3}$ \\
0.2 & 1.058 & 0.895 & -1.085 & 3.177 & 0.054 & $W_{4}>W_{1}>W_{2}>W_{5}>W_{3}$ \\
0.3 & 0.912 & 0.729 & -1.023 & 2.932 & -0.024 & $W_{4}>W_{1}>W_{2}>W_{5}>W_{3}$ \\
0.4 & 0.802 & 0.612 & -0.965 & 2.724 & -0.070 & $W_{4}>W_{1}>W_{2}>W_{5}>W_{3}$ \\
0.5 & 0.717 & 0.526 & -0.912 & 2.545 & -0.098 & $W_{4}>W_{1}>W_{2}>W_{5}>W_{3}$ \\
0.6 & 0.649 & 0.459 & -0.864 & 2.390 & -0.116 & $W_{4}>W_{1}>W_{2}>W_{5}>W_{3}$ \\
0.7 & 0.593 & 0.407 & -0.821 & 2.253 & -0.127 & $W_{4}>W_{1}>W_{2}>W_{5}>W_{3}$ \\
0.8 & 0.546 & 0.365 & -0.781 & 2.133 & -0.134 & $W_{4}>W_{1}>W_{2}>W_{5}>W_{3}$ \\
0.9 & 0.507 & 0.330 & -0.746 & 2.025 & -0.138 & $W_{4}>W_{1}>W_{2}>W_{5}>W_{3}$ \\
1 & 0.473 & 0.301 & -0.713 & 1.928 & -0.140 & $W_{4}>W_{1}>W_{2}>W_{5}>W_{3}$ \\
\hline
\end{tabular}



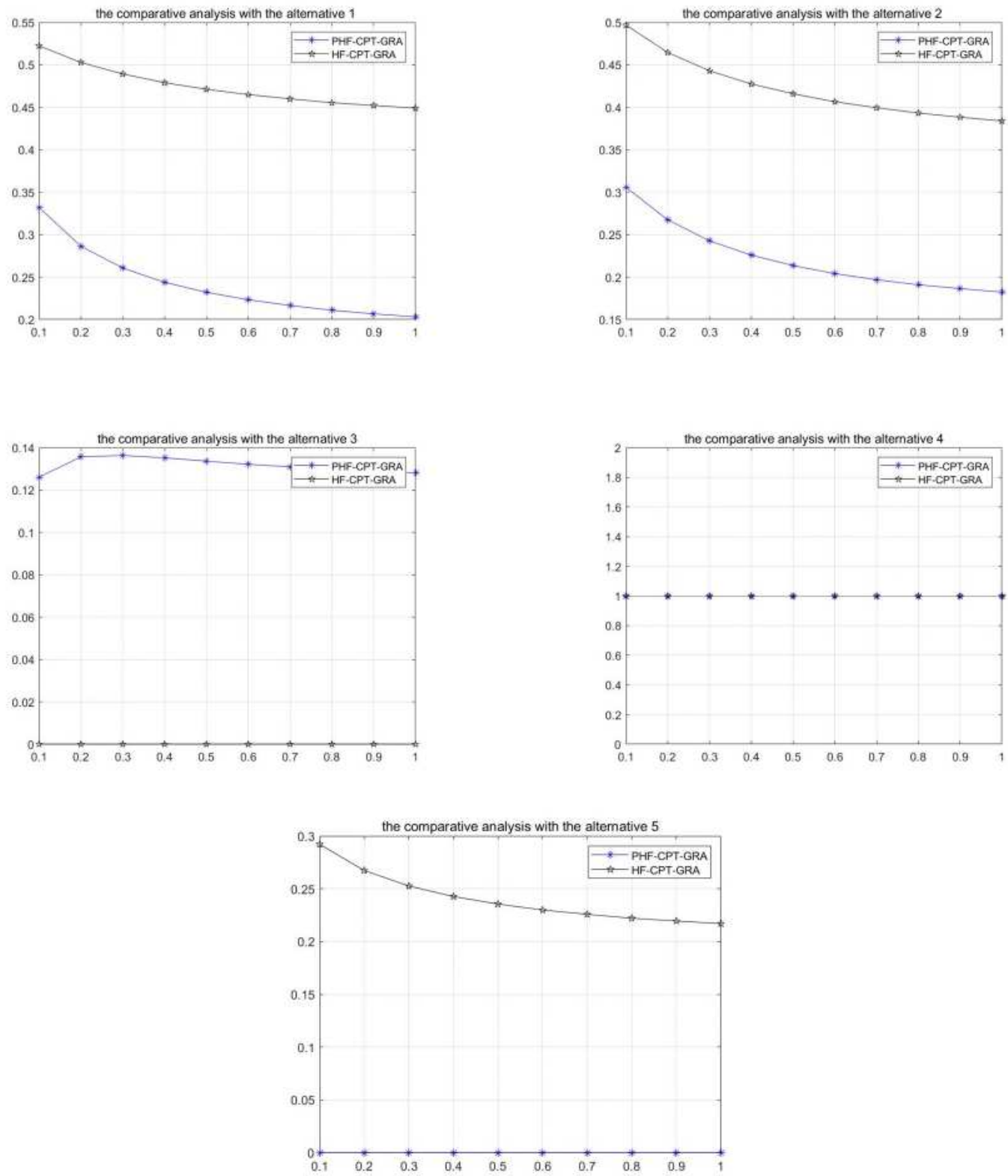

Figure 3. sensitive analysis of the extended GRA $0<\rho \leq 1, \lambda=2.25, \alpha=0.88, \beta=0.88$

Figure 3 shows that with the change of the value of $\rho$, the range of fluctuation obtained by extended GRA method with probabilistic hesitant fuzzy information is bigger than the one obtained by extended GRA method with hesitant fuzzy information. Among them, the comparison of the alternative 1 and 2 shows that the extended GRA method in different types of environment has the same trend and they all decrease with increasing the value of $\rho$.

In summary, the results in Figure 2 and 3 show that the extended GRA based on CPT in probabilistic hesitant fuzzy circumstance has a fluctuant stability under different schemes by comparing with the classical GRA method in the same environment. The Figure 3 shows us that 
compared with hesitant fuzzy environment, the probabilistic hesitant fuzzy environment contains more information and is more stable.

\subsection{Simulation Analysis}

The sensitive analysis performed above is based on a sample analysis, and there we use MTLAB software as a tool,1,000 samples were randomly generated for analysis. The ranking results are shown in Table 25 and Figure 4 to 8.

The data of Table 25 shows that in the probabilistic hesitant fuzzy circumstance compared with TODIM method, there are 688 out of 1,000 random samples have the same optimal results. Compared with TOPSIS method, there are 799 out of 1,000 random samples have the same results, while the comparison with PHFWA and PHFWG shows there are 515 and 466 sets out of 1,000 sets have the same sort of results.

Table 25. The ranking results of the method with 1000 sets of random samples

\begin{tabular}{ccc}
\hline Methods & $\begin{array}{c}\text { The value of the same } \\
\text { optimal results }\end{array}$ & $\begin{array}{c}\text { The value of the different } \\
\text { optimal result }\end{array}$ \\
\hline $\begin{array}{c}\text { PHF-CPT-GRA } \\
\text { PHF-TODIM[50] } \\
\text { PHF-CPT-GRA }\end{array}$ & 688 & 312 \\
PHF-TOPSIS[51] & 799 & 201 \\
PHF-CPT-GRA & & 485 \\
PHFWA[53] & 515 & \\
PHF-CPT-GRA & & 534 \\
PHFWG[53] & 466 & \\
\hline
\end{tabular}




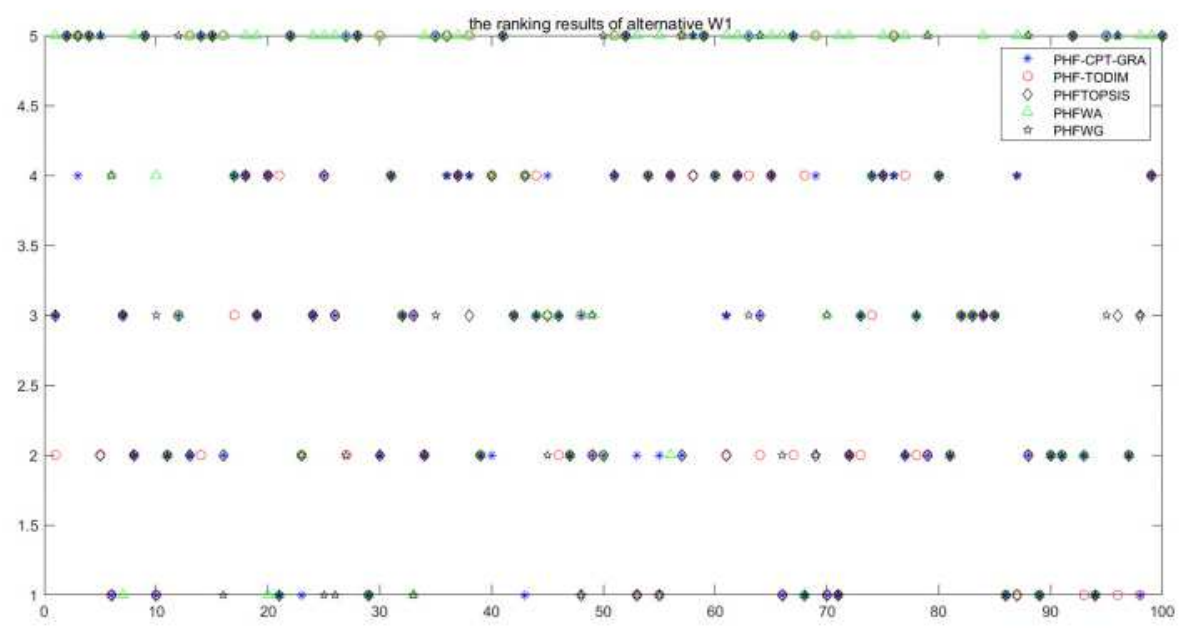

Figure 4. the ranking results of the alternative W1

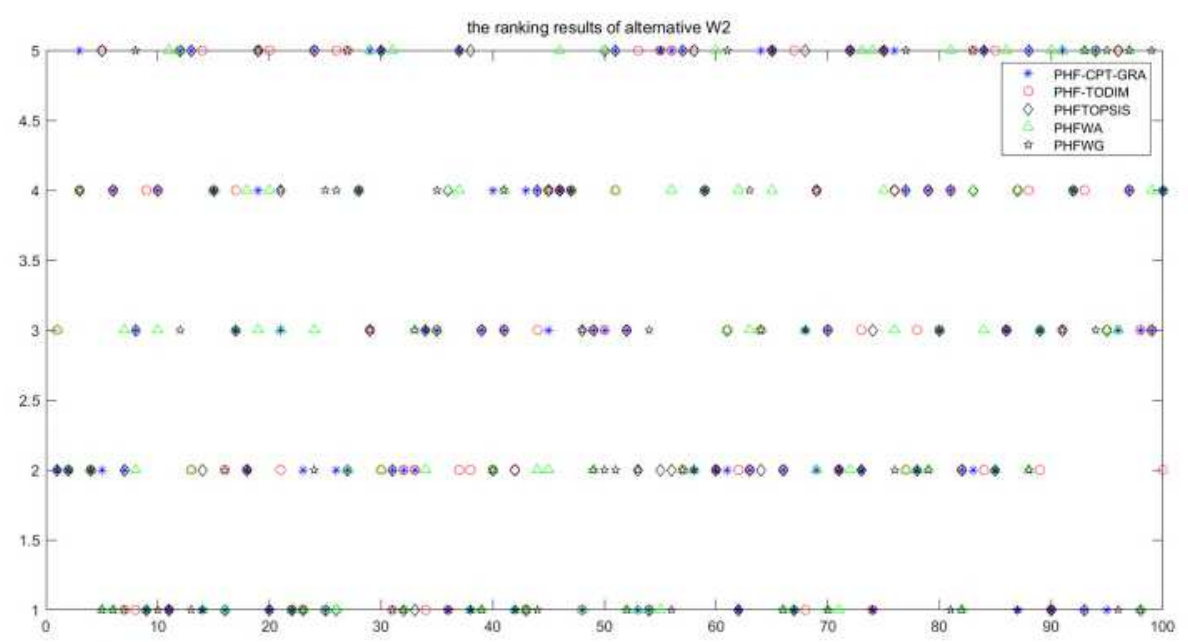

Figure 5. the ranking results of the alternative W2

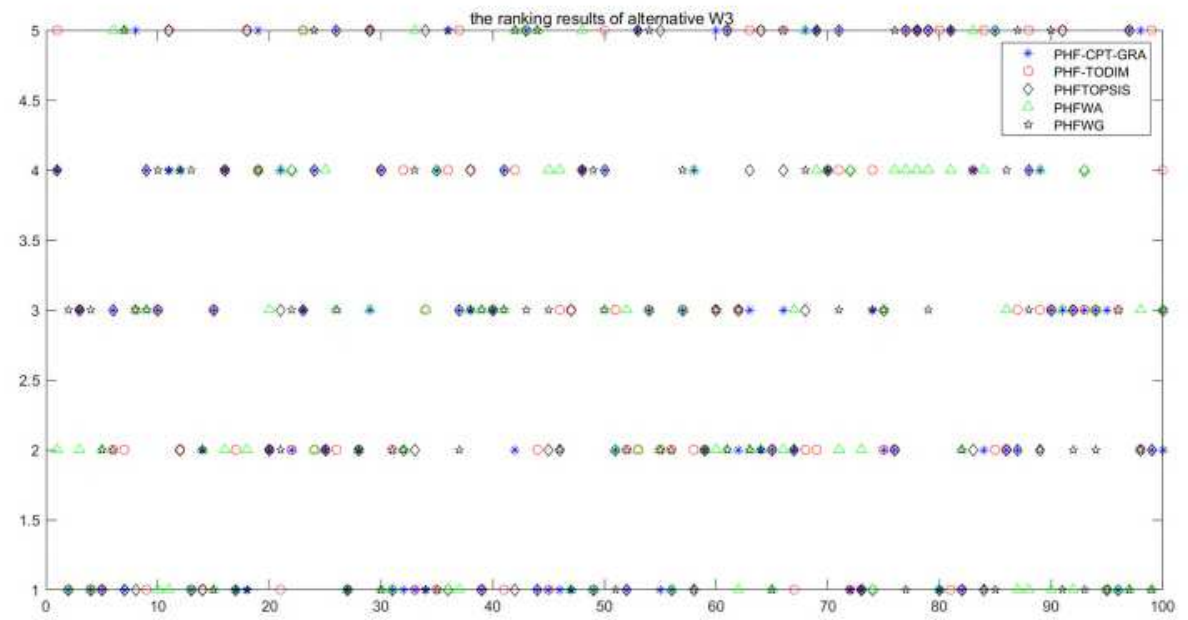

Figure 6. the ranking results of the alternative W3 


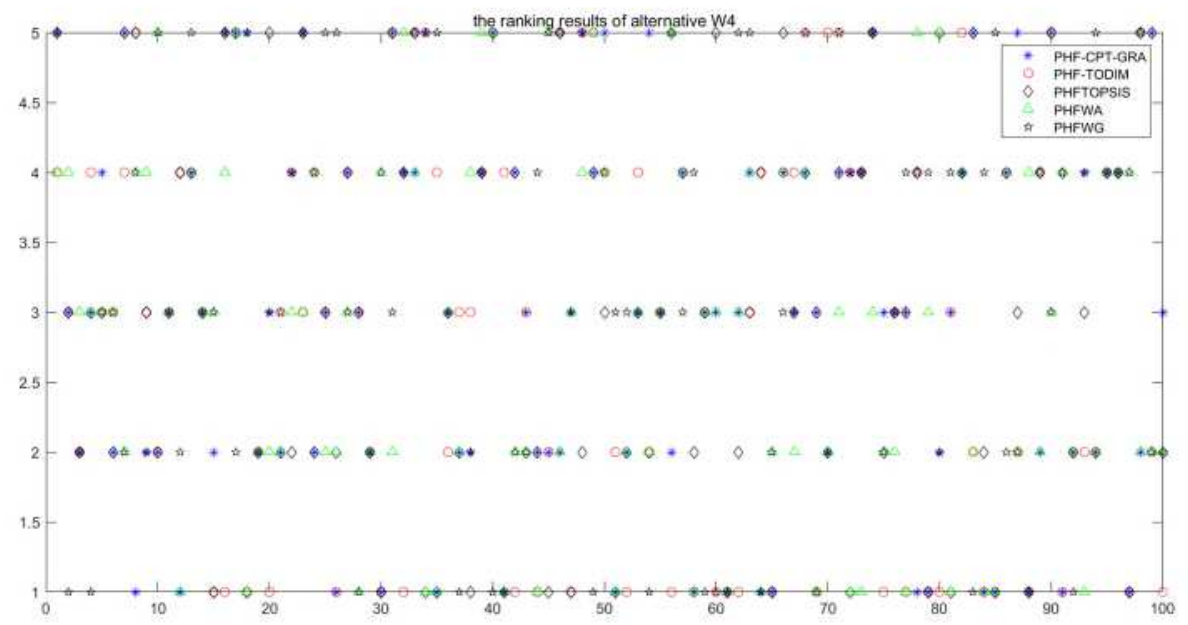

Figure 7. the ranking results of the alternative $\mathrm{W} 4$

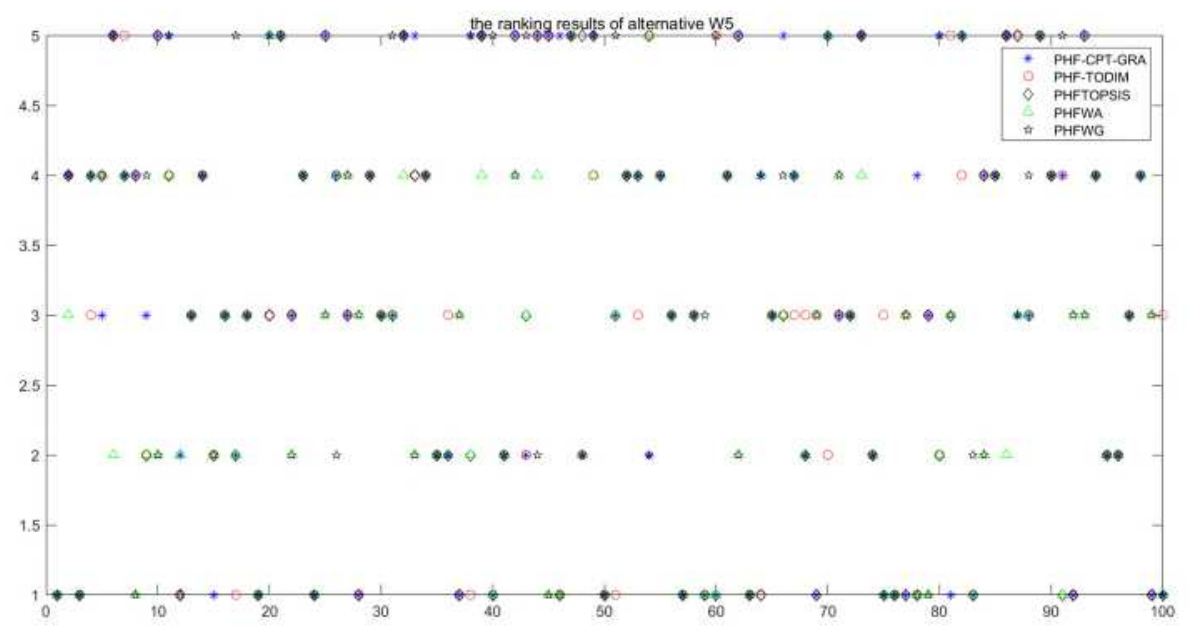

Figure 8. The ranking results of the alternative W5

With the calculation of a mass of random samples, the results which has a lot of same ranking results show us the applicability of extended GRA method base on cumulative prospect theory. While the different results by comparing with the other four methods show us that these methods have some differences because of their different priorities when making decisions.

\section{Conclusions}

The GRA, a method to measure the degree of correlation between factors according to the degree of similarity or difference between the development trends of factors is applied in complex environment with unknown information, while it ignores the psychological factors which may affect the results of the decision-making. Meanwhile, As the development of the decision-making, the complexity of the environment is increasing and fitting. Thus, in this paper, we developed classical 
GRA method with the cumulative prospect theory in probabilistic hesitant fuzzy environment (PHFCPT-GRA). What's more, we use the entropy method to obtain the weighting vector of each attributes. To testify the feasibility and stability of newly proposed method, we use four different methods to compare with it by using the sensitivity analysis and simulation analysis with the MATLAB software. The advantages of PHF-CPT-GRA method are as follows:

(1) It can help DMs make as accurate decisions as possible in the complex probabilistic hesitant fuzzy circumstance.

(2) It takes into account the psychology of DMs and combines classical GRA method and cumulative prospect theory considering the different propensity of DMs to risk.

In view of the above analysis, in the future, we firmly believe that PHF-CPT-GRA method will be applied in the more fields. Meanwhile, the integration of other classical method and CPT with more complex and uncertain information also needs more attention.

Authors' contribution Ningna Liao, Guiwu Wei and Xudong Chen discussed and constructed the mathematical models, found their applications and wrote the paper together.

\section{Compliance with ethical standards}

\section{Ethical approval}

This article does not contain any studies with human participants or animals performed by any of the authors.

\section{Conflict of interest}

The authors declare that they have no conflict of interest.

\section{Data Availability}

The data used to support the findings of this study are included in the article.

\section{Reference}

[1] D.F. Li, S.P. Wan, Fuzzy heterogeneous multiattribute decision making method for outsourcing provider selection, Expert Systems with Applications, 41 (2014) 3047-3059.

[2] G.F. Yu, D.F. Li, W. Fei, A novel method for heterogeneous multi-attribute group decision making 
with preference deviation, Computers \& Industrial Engineering, 124 (2018) 58-64.

[3] P.D. Liu, A weighted aggregation operators multi-attribute group decision-making method based on interval-valued trapezoidal fuzzy numbers, Expert Systems with Applications, 38 (2011) 1053-1060.

[4] P.D. Liu, S.M. Chen, Group Decision Making Based on Heronian Aggregation Operators of Intuitionistic Fuzzy Numbers, Ieee Transactions on Cybernetics, 47 (2017) 2514-2530.

[5] G. Wei, J. Wu, Y. Guo, J. Wang, C. Wei, An extended COPRAS model for multiple attribute group decision making based on single-valued neutrosophic 2-tuple linguistic environment, Technological and Economic Development of Economy, 27 (2021) 353-368.

[6] S. Opricovic, G.H. Tzeng, Compromise solution by MCDM methods: A comparative analysis of VIKOR and TOPSIS, European Journal of Operational Research, 156 (2004) 445-455.

[7] T.T. He, G.W. Wei, R. Lin, J.P. Lu, C. Wei, J. Wu, Pythagorean interval 2-tuple linguistic VIKOR method for evaluating human factors in construction project management, Iranian Journal of Fuzzy Systems, 17 (2020) 93-105.

[8] G.W. Wei, J. Wang, J.P. Lu, J. Wu, C. Wei, F.E. Alsaadi, T. Hayat, VIKOR method for multiple criteria group decision making under 2-tuple linguistic neutrosophic environment, Economic ResearchEkonomska Istrazivanja, 33 (2020) 3185-3208.

[9] D. Pamucar, G. Cirovic, The selection of transport and handling resources in logistics centers using Multi-Attributive Border Approximation area Comparison (MABAC), Expert Systems with Applications, 42 (2015) 3016-3028.

[10] J. Wang, G.W. Wei, C. Wei, Y. Wei, MABAC method for multiple attribute group decision making under q-rung orthopair fuzzy environment, Defence Technology, 16 (2020) 208-216.

[11] G.W. Wei, Y. He, F. Lei, J. Wu, C. Wei, MABAC method for multiple attribute group decision making with probabilistic uncertain linguistic information, Journal of Intelligent \& Fuzzy Systems, 39 (2020) 3315-3327.

[12] G.W. Wei, Y. He, F. Lei, J. Wu, C. Wei, Y.F. Guo, Green supplier selection with an uncertain probabilistic linguistic MABAC method, Journal of Intelligent \& Fuzzy Systems, 39 (2020) 31253136.

[13] M. Keshavarz Ghorabaee, E.K. Zavadskas, Z. Turskis, J. Antucheviciene, A new combinative distance-based assessment (CODAS) method for multi-criteria decision-making, Economic Computation and Economic Cybernetics Studies and Research, 50 (2016) 25-44. 
[14] T. He, S. Zhang, G. Wei, R. Wang, J. Wu, C. Wei, CODAS method for 2-tuple linguistic pythagorean fuzzy multiple attribute group decision making and its application to financial management performance assessment, Technological and Economic Development of Economy, 26 (2020) 920932.

[15] J. Lan, J. Wu, Y. Guo, C. Wei, G. Wei, H. Gao, CODAS methods for multiple attribute group decision making with interval-valued bipolar uncertain linguistic information and their application to risk assessment of Chinese enterprises' overseas mergers and acquisitions, Economic ResearchEkonomska Istraživanja, (2021) https://doi.org/10.1080/1331677X.1332020.1868323.

[16] C. Wei, J. Wu, Y. Guo, G. Wei, Green supplier selection based on CODAS method in probabilistic uncertain linguistic environment, Technological and Economic Development of Economy, (2021) https://doi.org/10.3846/tede.2021.14078.

[17] M. Keshavarz Ghorabaee, E.K. Zavadskas, L. Olfat, Z. Turskis, Multi-Criteria Inventory Classification Using a New Method of Evaluation Based on Distance from Average Solution (EDAS), Informatica, 26 (2015) 435-451.

[18] Y. He, F. Lei, G.W. Wei, R. Wang, J. Wu, C. Wei, EDAS method for multiple attribute group decision making with probabilistic uncertain linguistic information and its application to green supplier selection, International Journal of Computational Intelligence Systems, 12 (2019) 1361-1370.

[19] N. Kundakci, An integrated method using MACBETH and EDAS methods for evaluating steam boiler alternatives, Journal of Multi-Criteria Decision Analysis, 26 (2019) 27-34.

[20] J. Ouenniche, O.J.U. Perez, A. Ettouhami, A new EDAS-based in-sample-out-of-sample classifier for risk-class prediction, Management Decision, 57 (2019) 314-323.

[21] D. Schitea, M. Deveci, M. Iordache, K. Bilgili, I.Z. Akyurt, I. Iordache, Hydrogen mobility roll-up site selection using intuitionistic fuzzy sets based WASPAS, COPRAS and EDAS, International Journal of Hydrogen Energy, 44 (2019) 8585-8600.

[22] T. He, G. Wei, J. Lu, J. Wu, C. Wei, Y. Guo, A novel EDAS based method for multiple attribute group decision making with pythagorean 2-tuple linguistic information, Technological and Economic Development of Economy, 26 (2020) 1125-1138.

[23] W.K.M. Brauers, E.K. Zavadskas, The MOORA method and its application to privatization in a transition economy, Control and Cybernetics, 35 (2006) 445-469.

[24] H.C. Liu, J.X. You, C. Lu, M.M. Shan, Application of interval 2-tuple linguistic MULTIMOORA 
method for health-care waste treatment technology evaluation and selection, Waste Management, 34 (2014) 2355-2364.

[25] E.K. Zavadskas, J. Antucheviciene, S.H.R. Hajiagha, S.S. Hashemi, The Interval-Valued Intuitionistic Fuzzy MULTIMOORA Method for Group Decision Making in Engineering, Mathematical Problems in Engineering, (2015).

[26] A. Hafezalkotob, A. Hafezalkotob, Fuzzy entropy-weighted MULTIMOORA method for materials selection, Journal of Intelligent \& Fuzzy Systems, 31 (2016) 1211-1226.

[27] L. Gomes, L.A.D. Rangel, An application of the TODIM method to the multicriteria rental evaluation of residential properties, European Journal of Operational Research, 193 (2009) 204-211.

[28] M.W. Zhao, G.W. Wei, C. Wei, J. Wu, Pythagorean Fuzzy TODIM Method Based on the Cumulative Prospect Theory for MAGDM and Its Application on Risk Assessment of Science and Technology Projects, International Journal of Fuzzy Systems, (2021) https://doi.org/10.1007/s40815-4002000986-40818.

[29] M. Zhao, G. Wei, C. Wei, Y. Guo, CPT - TODIM method for bipolar fuzzy multi - attribute group decision making and its application to network security service provider selection, International Journal of Intelligent Systems, 36 (2021) 1943-1969.

[30] Z. Jiang, G. Wei, J. Wu, X. Chen, CPT-TODIM method for picture fuzzy multiple attribute group decision making and its application to food enterprise quality credit evaluation, Journal of Intelligent \& Fuzzy Systems, 4 (2021) 10115-10128.

[31] T.T. He, G.W. Wei, J.P. Lu, C. Wei, R. Lin, Pythagorean 2-tuple linguistic Taxonomy method for supplier selection in medical instrument industries, International Journal of Environmental Research and Public Health, 16 (2019) 4875.

[32] L. Xiao, S. Zhang, G. Wei, J. Wu, C. Wei, Y. Guo, Y. Wei, Green supplier selection in steel industry with intuitionistic fuzzy Taxonomy method, Journal of Intelligent \& Fuzzy Systems, 39 (2020) 72477258.

[33] J.L. Deng, Introduction to Grey System, The Journal of Grey System, 1 (1989) 1-24.

[34] Y. Kuo, T. Yang, G.W. Huang, The use of grey relational analysis in solving multiple attribute decision-making problems, Computers \& Industrial Engineering, 55 (2008) 80-93.

[35] B.Z. Zhu, L.L. Yuan, S.X. Ye, Examining the multi-timescales of European carbon market with grey relational analysis and empirical mode decomposition, Physica a-Statistical Mechanics and Its 
Applications, 517 (2019) 392-399.

[36] J. Tang, H.L. Zhu, Z. Liu, F. Jia, X.X. Zheng, Urban Sustainability Evaluation under the Modified TOPSIS Based on Grey Relational Analysis, International Journal of Environmental Research and Public Health, 16 (2019).

[37] Y.S. Tan, H. Chen, S. Wu, Evaluation and Implementation of Environmentally Conscious Product Design by Using AHP and Grey Relational Analysis Approaches, Ekoloji, 28 (2019) 857-864.

[38] H.J. Liu, Q.Y. Liu, Y.R. Hu, Evaluating Risks of Mergers \& Acquisitions by Grey Relational Analysis Based on Interval-Valued Intuitionistic Fuzzy Information, Mathematical Problems in Engineering, (2019).

[39] A. Tversky, D. Kahneman, Advances in prospect theory: cumulative representation of uncertainty, Journal of Risk and Uncertainty, 5 (1992) 297-323.

[40] L.A. Zadeh, Fuzzy Sets, in: Information and Control, 1965, pp. 338-356.

[41] K.T. Atanassov, Intuitionistic fuzzy sets, Fuzzy Sets and Systems, 20 (1986) 87-96.

[42] V. Torra, Hesitant Fuzzy Sets, International Journal of Intelligent Systems, 25 (2010) 529-539.

[43] Z.S. Xu, W. Zhou, Consensus building with a group of decision makers under the hesitant probabilistic fuzzy environment, Fuzzy Optimization and Decision Making, 16 (2017) 481-503.

[44] J. Ding, Z.S. Xu, N. Zhao, An interactive approach to probabilistic hesitant fuzzy multi-attribute group decision making with incomplete weight information, Journal of Intelligent \& Fuzzy Systems, 32 (2017) 2523-2536.

[45] J. Li, Q.X. Chen, L.L. Niu, Z.X. Wang, An ORESTE approach for multi-criteria decision-making with probabilistic hesitant fuzzy information, International Journal of Machine Learning and Cybernetics, 11 (2020) 1591-1609.

[46] C.X. Yu, Y.F. Shao, K. Wang, L.P. Zhang, A group decision making sustainable supplier selection approach using extended TOPSIS under interval-valued Pythagorean fuzzy environment, Expert Systems with Applications, 121 (2019) 1-17.

[47] H.D. Wang, J.L. Yao, J. Yan, M.G. Dong, An Extended TOPSIS Method Based on Gaussian Interval Type-2 Fuzzy Set, International Journal of Fuzzy Systems, 21 (2019) 1831-1843.

[48] L. Perez-Dominguez, D. Luviano-Cruz, D. Valles-Rosales, J.I.H. Hernandez, M.I.R. Borbon, Hesitant Fuzzy Linguistic Term and TOPSIS to Assess Lean Performance, Applied Sciences-Basel, 9 (2019). 
[49] X. Sha, C. Yin, Z. Xu, S. Zhang, Probabilistic Hesitant Fuzzy TOPSIS emergency decision-making method based on the Cumulative Prospect Theory, Journal of Intelligent \& Fuzzy Systems, 40 (2021) 4367-4383.

[50] W.K. Zhang, J. Du, X.L. Tian, FINDING A PROMISING VENTURE CAPITAL PROJECT WITH TODIM UNDER PROBABILISTIC HESITANT FUZZY CIRCUMSTANCE, Technological and Economic Development of Economy, 24 (2018) 2026-2044.

[51] J. Wu, X.D. Liu, Z.W. Wang, S.T. Zhang, Dynamic emergency decision-making method with Probabilistic Hesitant Fuzzy information based on GM(1,1) and TOPSIS, Ieee Access, 7 (2019) 70547066.

[52] X.L. Tian, M.L. Niu, J.S. Ma, Z.S. Xu, A Novel TODIM with Probabilistic Hesitant Fuzzy Information and Its Application in Green Supplier Selection, Complexity, 2020 (2020) 26.

[53] J. Li, Z.X. Wang, Consensus Building for Probabilistic Hesitant Fuzzy Preference Relations with Expected Additive Consistency, International Journal of Fuzzy Systems, 20 (2018) 1495-1510. 